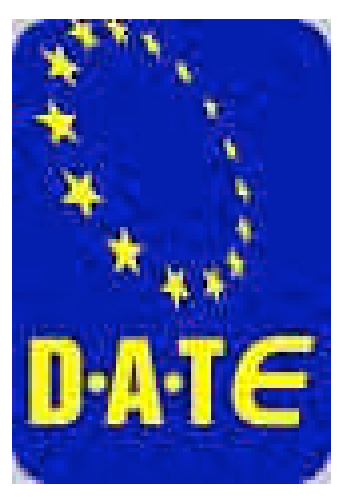

\title{
Designing Micro/Nano Systems for a safer and healthier tomorrow
}

Giovanni De Micheli

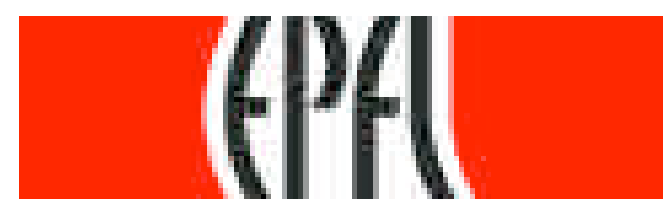

(icentres! 


\section{Quo vadis ?}

- We came a long way ...

- 50 years of electronics
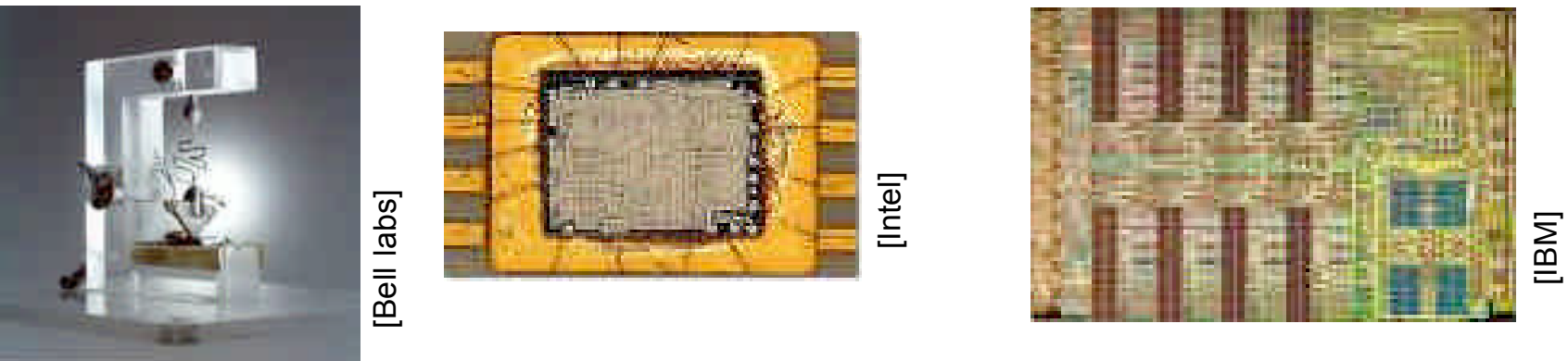

- ... and where are we going?

- The next 50 years
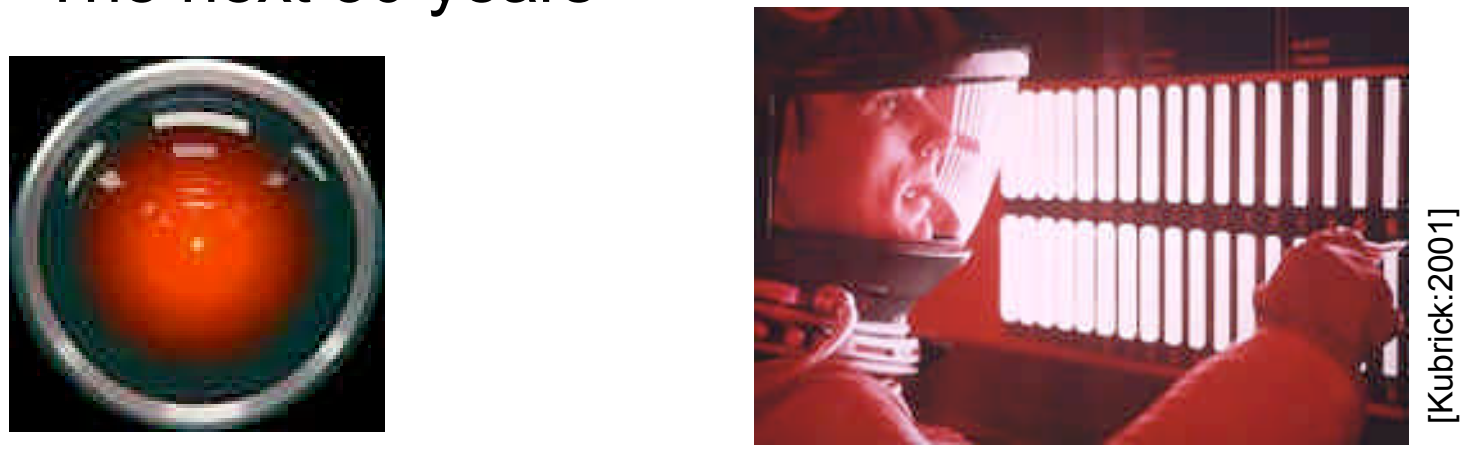

(c) Giovanni De Micheli 


\section{How did we affect society?}

- From transistor radio ...
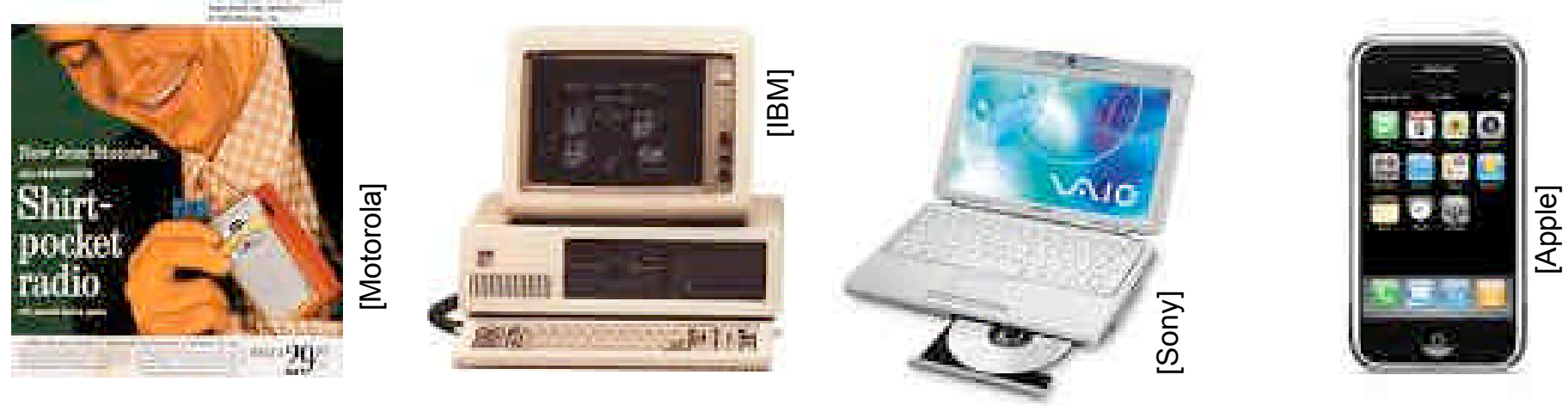

- Challenges:

- More processing power needed

- Less energy consumption desired

- What is the technology of the future?

- Can we have a deeper impact into society?

- Who will benefit?

- Socially?

- Economically? Which market sector? 


\section{How did we engineer products?}

- Electronic Design Automation (EDA)

- Provides us with the enabling technology

- Formal modeling, analysis, synthesis

- But EDA

- ... is entangled in solving deep submicron issues

- ... missed opportunities at system-level design

- ... is still a small niche market

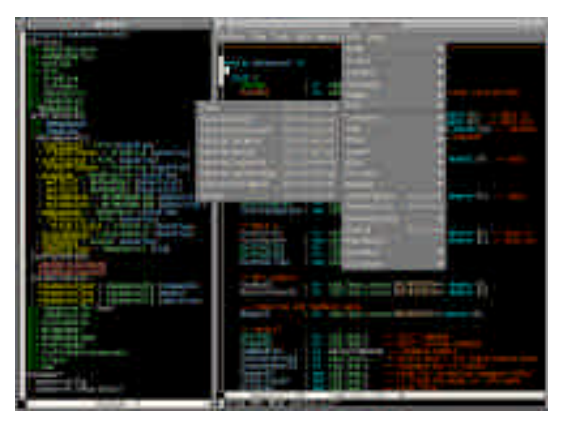

- Can we reposition DA as a central engineering task?

- Broader in scope

- Scientifically challenging

- Attracting the best young researchers

- Creating more value 


\section{The next fifty years...}

- Ubiquitously-distributed electronics

- Electronic circuits and systems distributed in clothing, car, home, office, environment...

- A global market affecting everybody's everyday's life

- Some audacious goals:

Break language barriers

Eliminate energy dependence

Link up every human

Better health, safety and longevity

Protect and monitor our environment

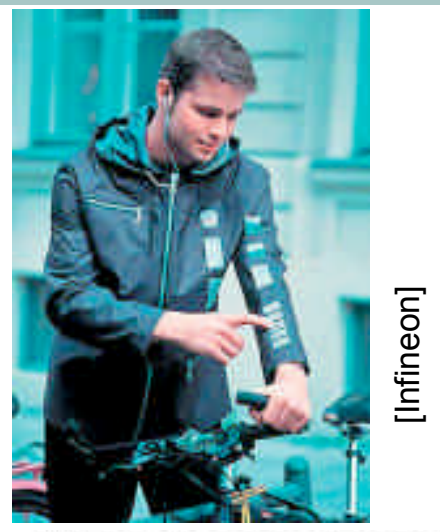

EIB: EEN SYSTEEM MET UITGEBREIDE MOGELIUKHEDEN

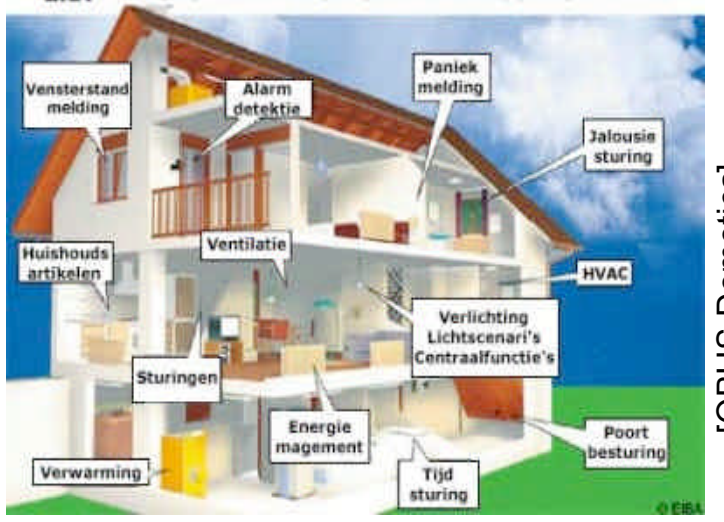




\section{The way ahead}

\section{Distributed systems}

Embedded systems on chip Heterogeneity, connectivity, human

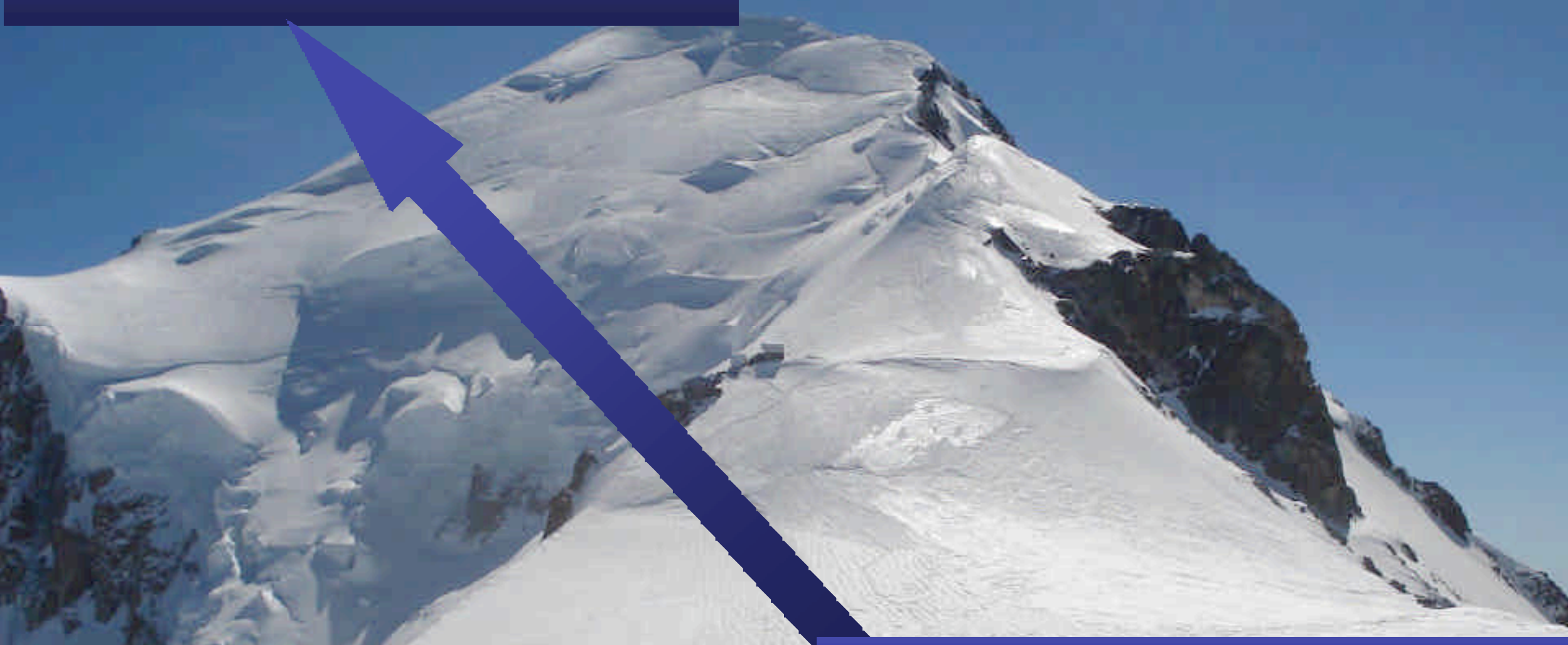

Systems on Chips

Technology, architectures, integration 


\section{Redefining electronic chip design}

- Requirements:
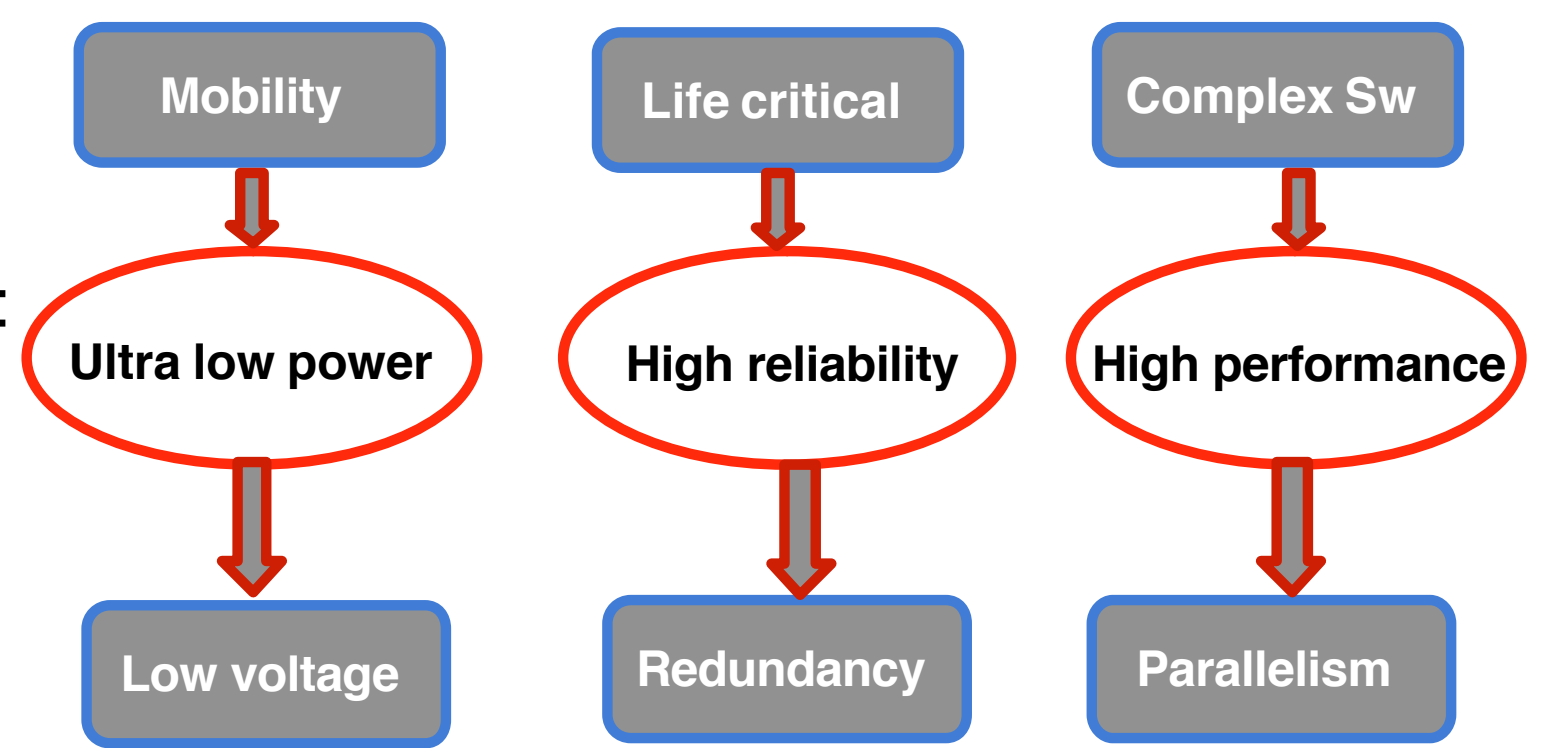

- From processors to multi-processors

- Technology support

- Systems and software redesign

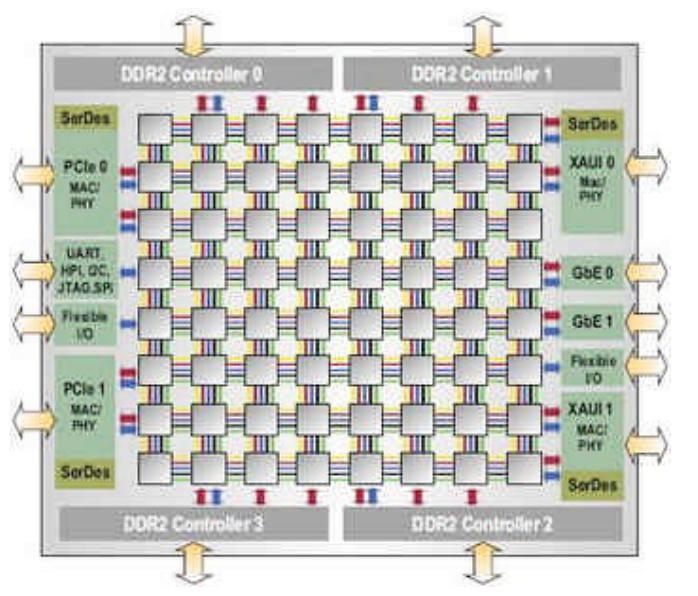




\section{The fabrication technology support}

- Beyond CMOS: a myriad of new ideas
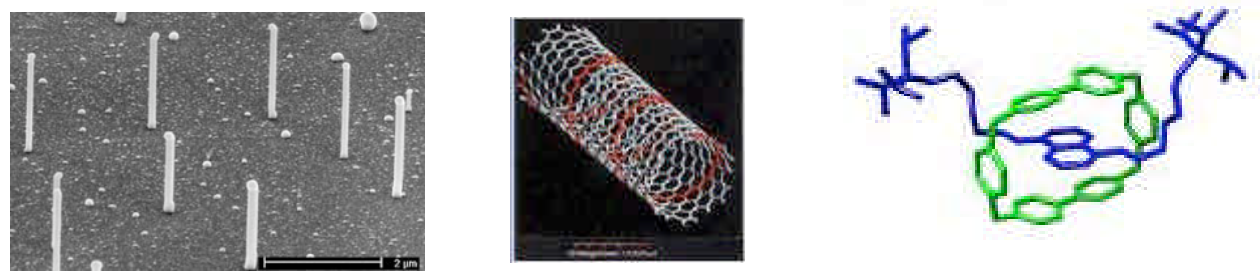

- Are these technologies apt/ready for system design?

- Can they mix and match with CMOS?
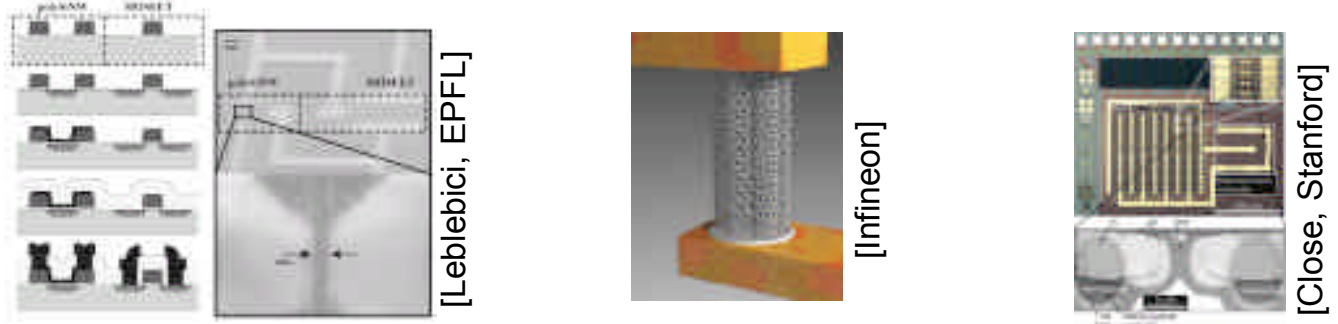

- How do we design with these technologies?

- Higher defect densities and failure rates 


\section{New computational structures}

- Computation requirements

- Predictable design

- Fast design closure

- Array based computation

- Matching nano and micro
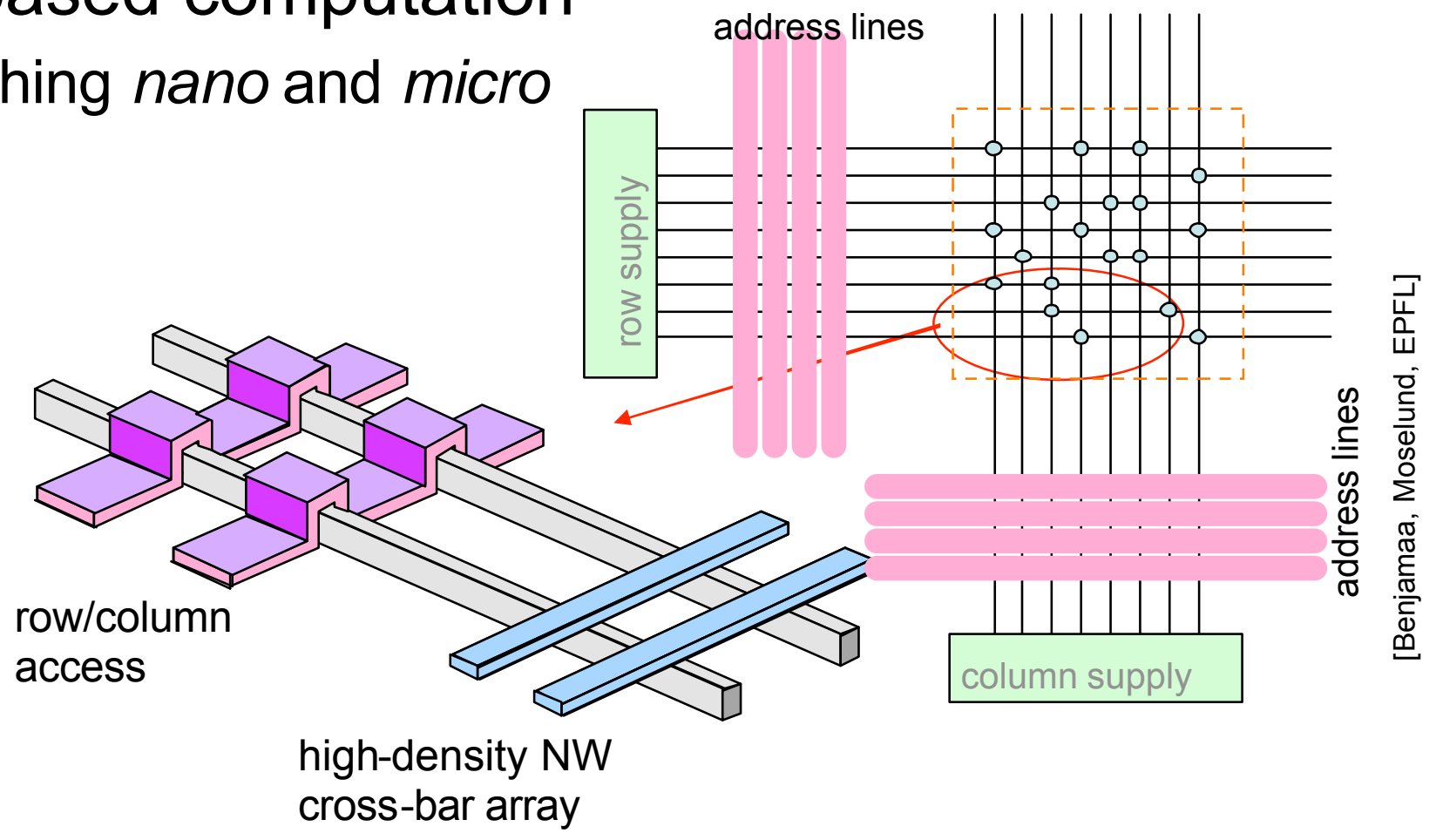

(c) Giovanni De Micheli 


\section{New communication structures}

- Design requirements:

- Predictable design

- Fast design closure

- Network on Chip communication

- Modular and flexible interconnect

- Reliable on-chip communication

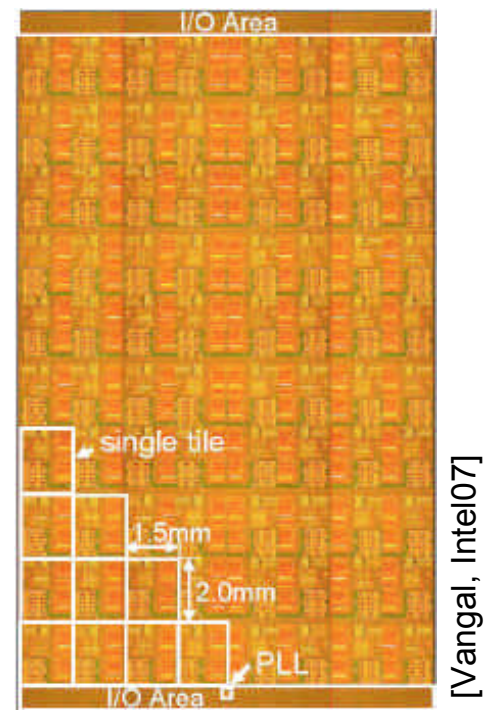

- Structured design with synthesis and optimization support
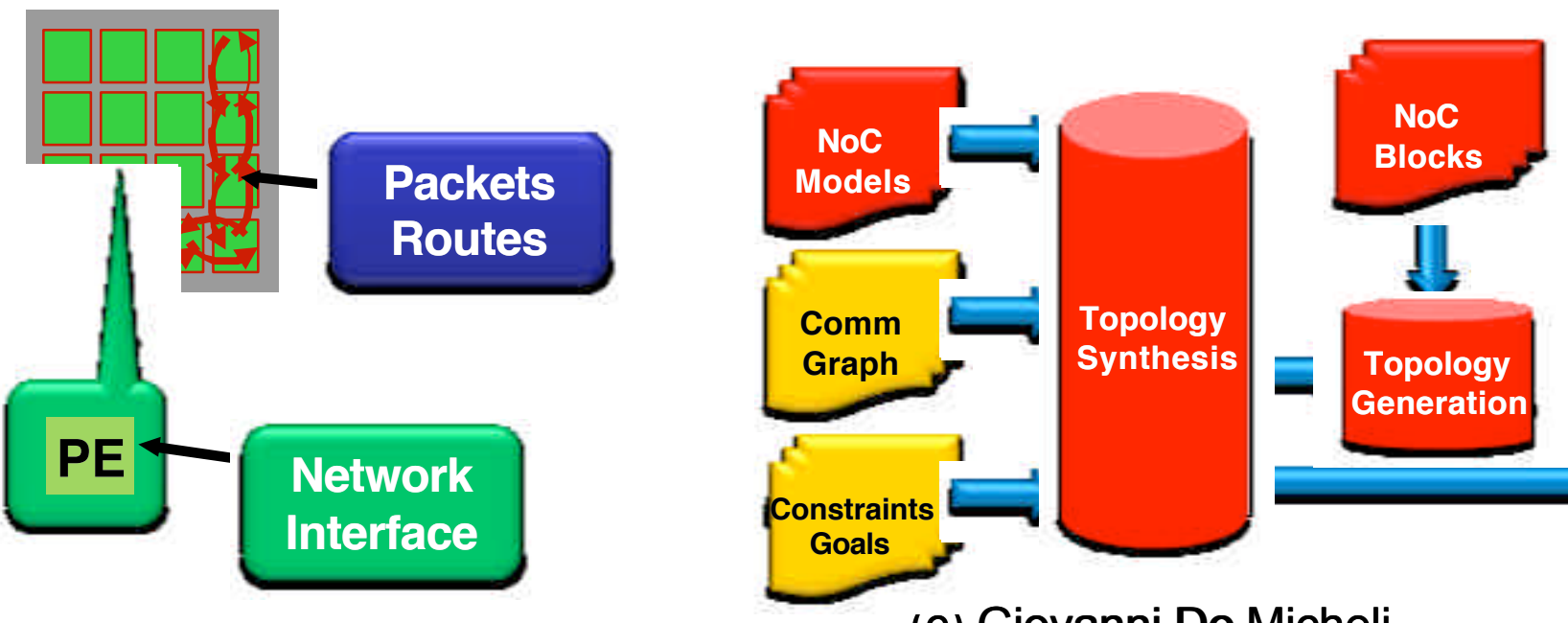

(C) Giovanni De Micheli

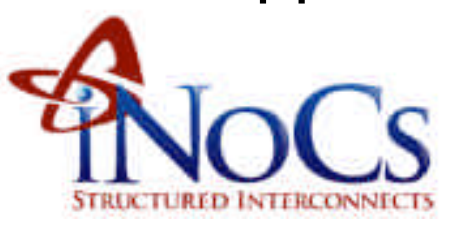




\section{New packaging technology}

- From planar to 3D integration

- Chips have limited wiring resources

- Electrical and manufacturing constraints limit heterogeneous planar integration

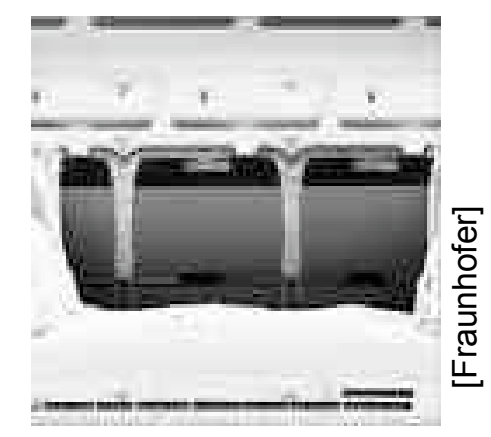

- Through silicon vias allow designer to stack:

- Computing arrays

- Memory arrays

- Analog and RF circuitry

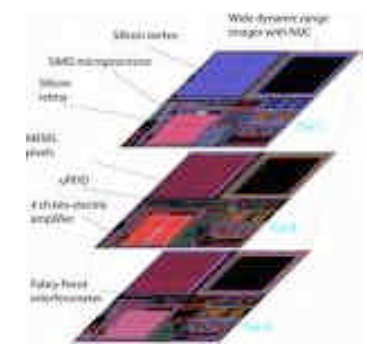

- 3D NoCs provide effective and reconfigurable means of realizing communication

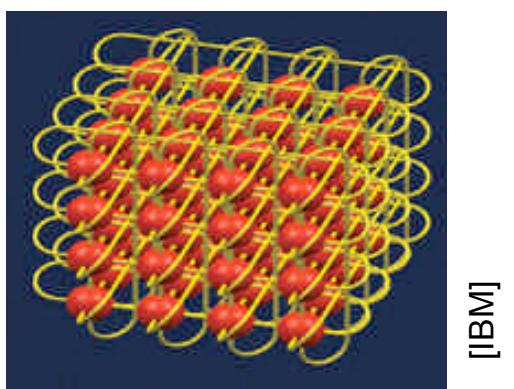




\section{Heterogeneous integration}

- Electrical and mechanical parts

- Microactuators, scavengers, microfluidics
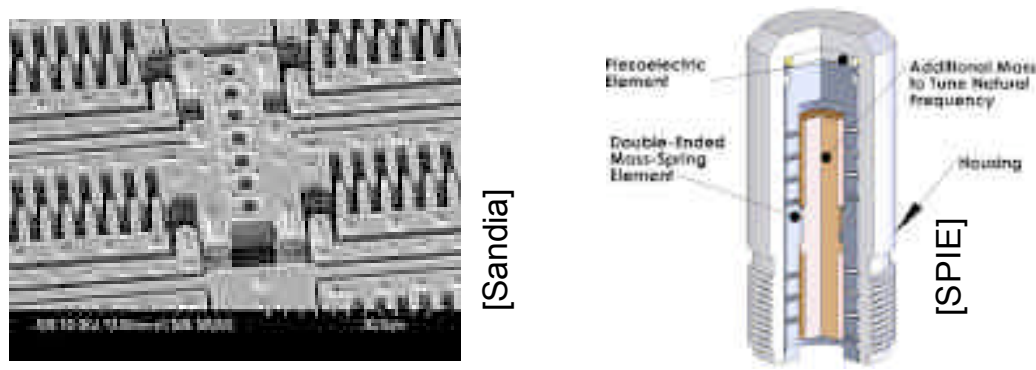

- Electronics meets the living world
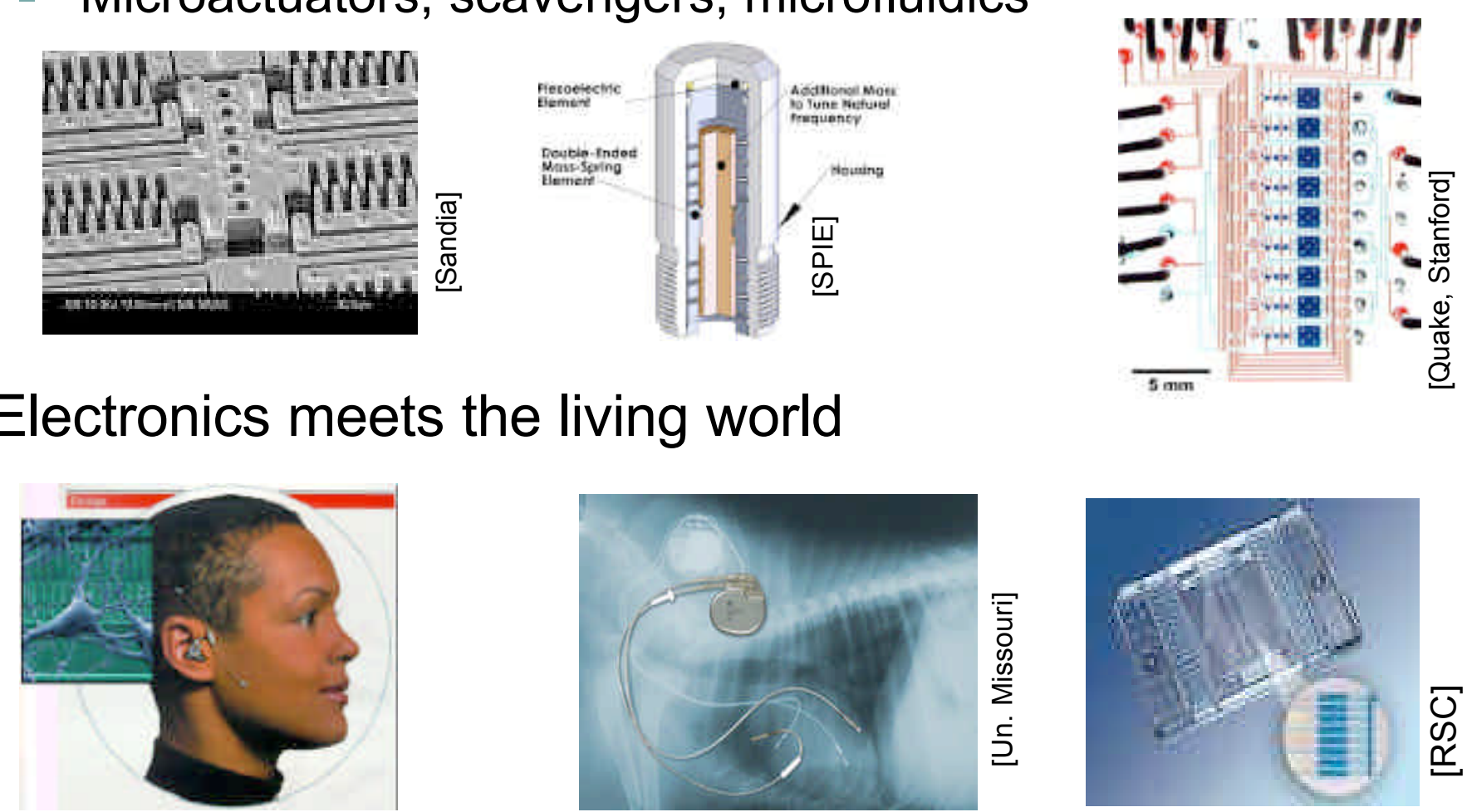

- Universal co-design

(c) Giovanni De Micheli 


\section{The micro and macro world}

- Chips embedded in environment

- Local sensing, processing and communicating
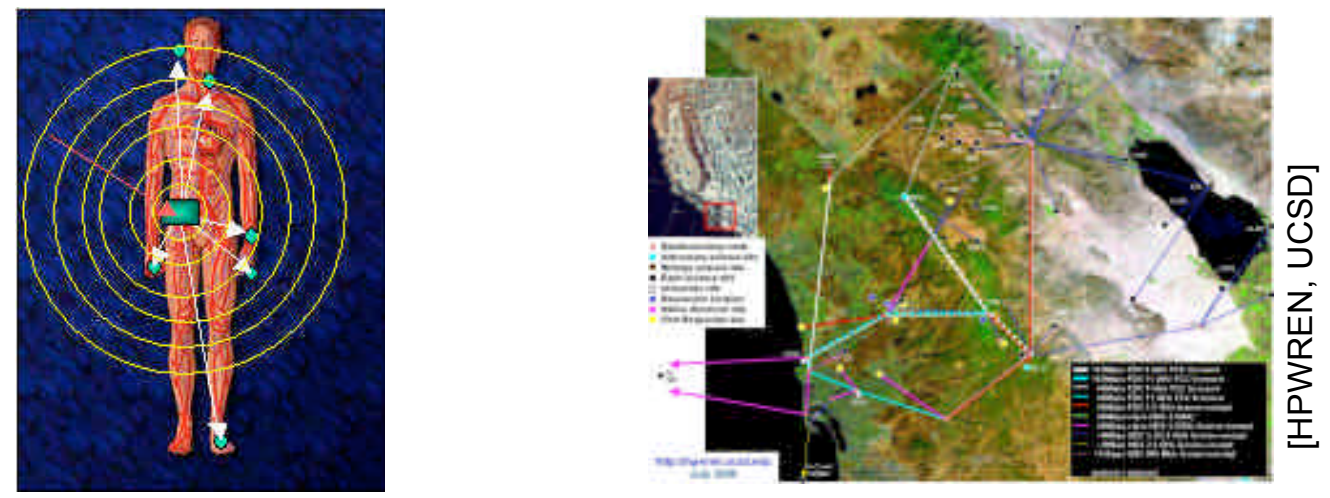

- Information production and consumption

- The challenge of effective, correct and dependable SW

- Avoiding system-level failure

- Safety-critical applications

- Application, system and communication SW 


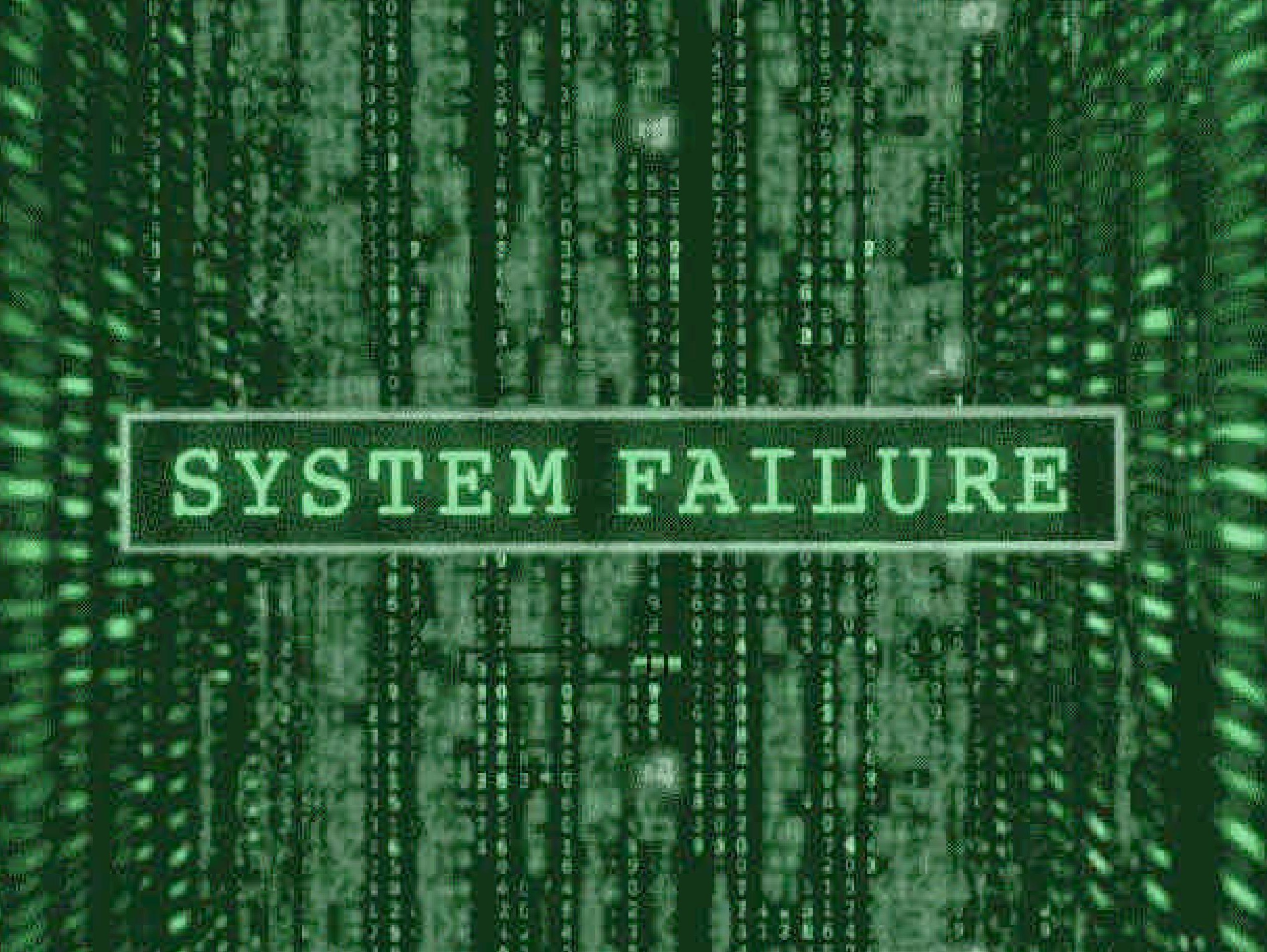




\section{The enabling design technology}

- System-level design technology

- Evolution of EDA

- Modeling, analysis, synthesis

- The discipline brought by EDA enabled very complex chips to be successfully designed and operated

- A bigger perspective

- How to engineer complex multivariate systems

- Address all aspects of embedded system design

- Scientific and commercial value stems from the systems aspect 


\section{DA Evolution \\ through three illustrative examples}

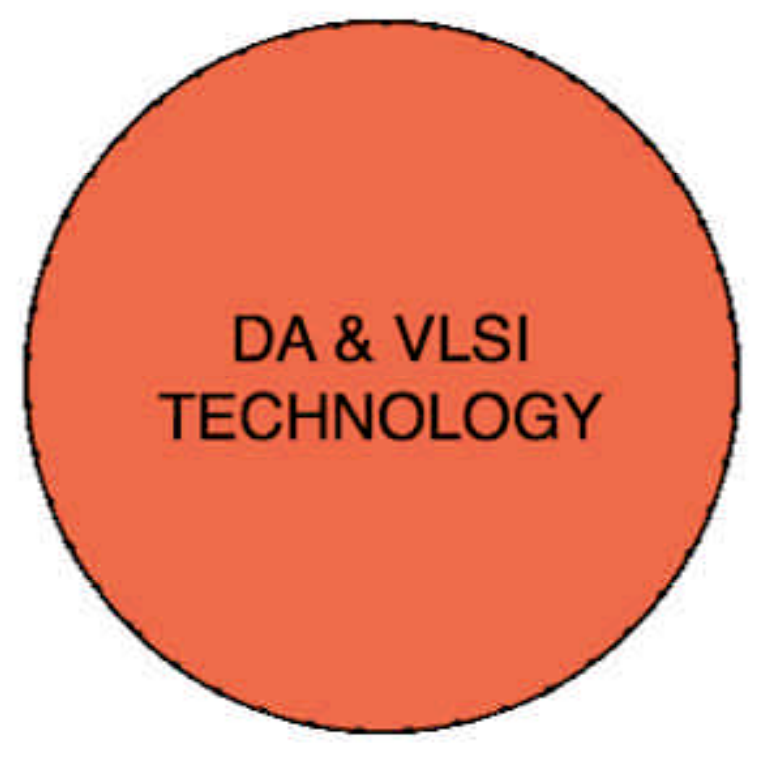

(c) Giovanni De Micheli 


\section{DA Evolution \\ through three illustrative examples}

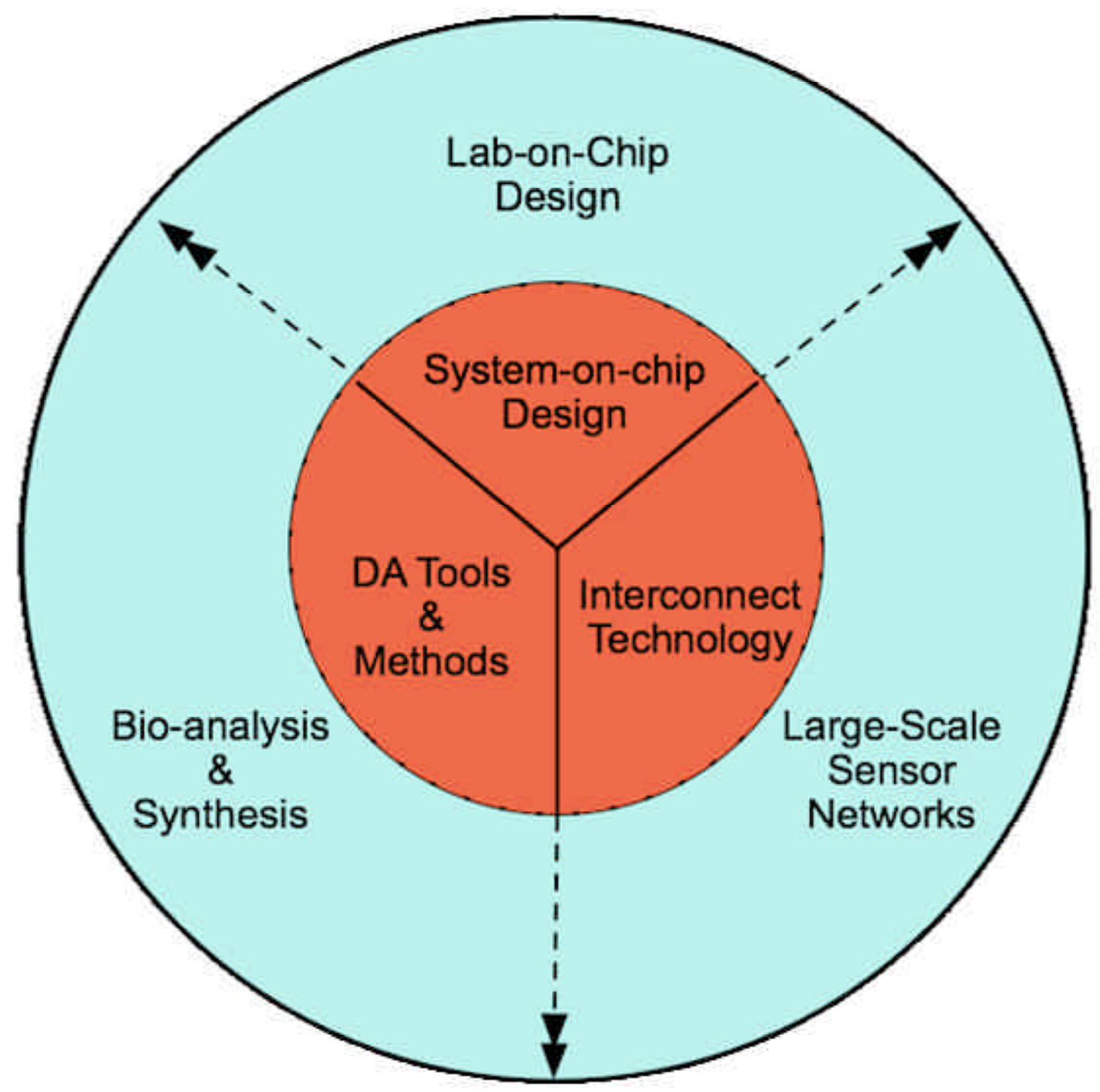




\section{Lab-on-chip technology}

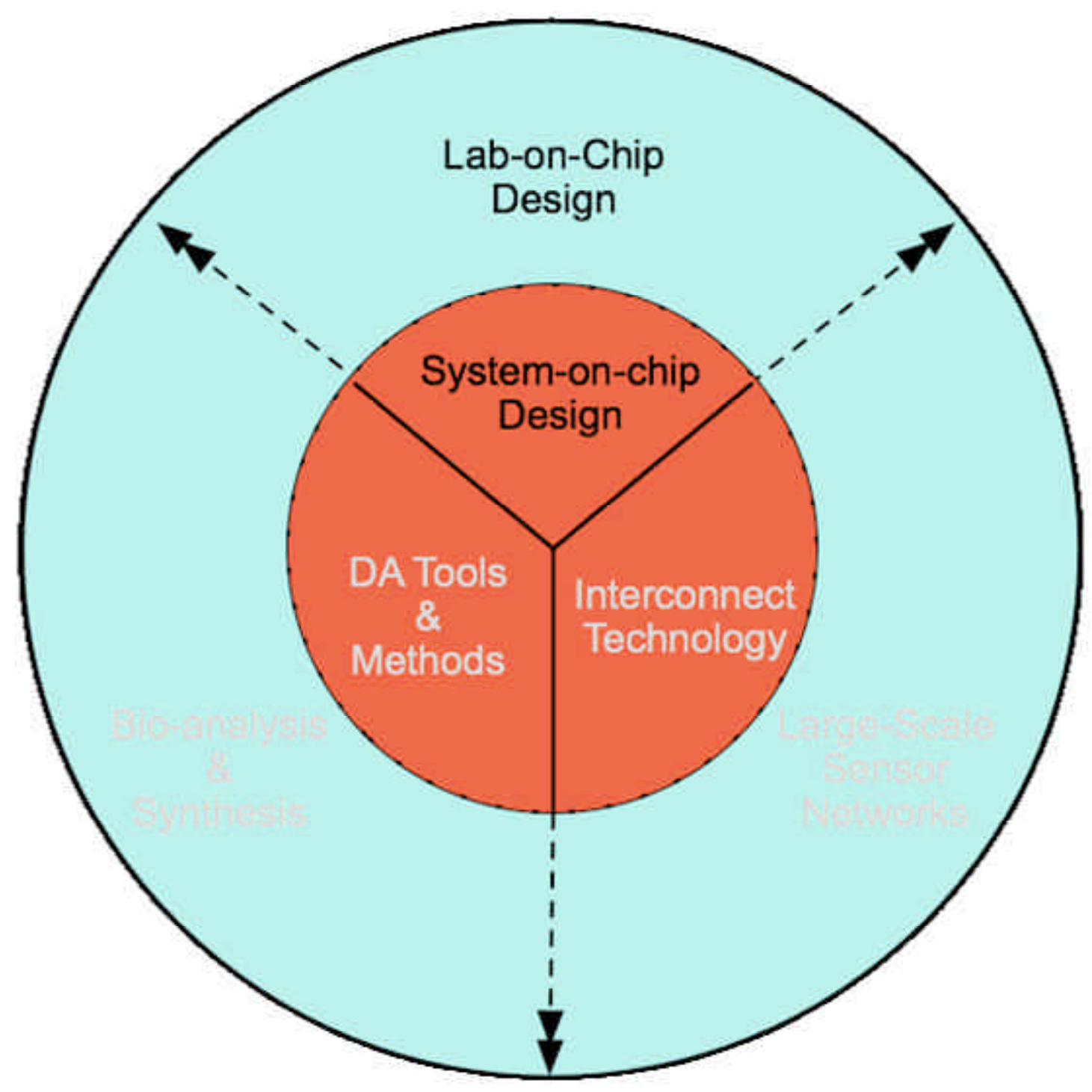




\section{Computer-aided diagnosis (CAD?)}

- Lab on chip at point of care

- Perform biochemical test on the field

- Faster, cheaper, more effective...

- How

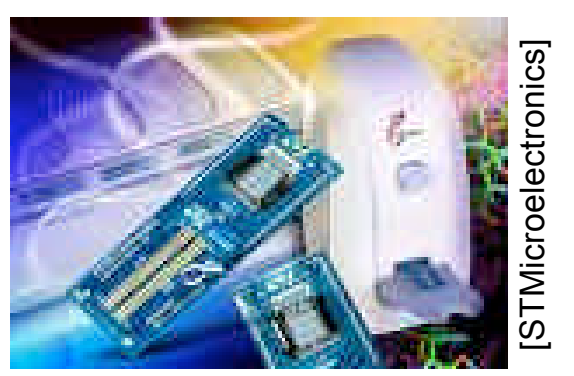

- The ultimate hybridization of technologies:

- Microfluidic: sample transport

- Sensors: binding proteins, DNA to probes

- Low-noise electronics

- Powerful data processing algorithms and software

- The promise of lab on chip is to revolutionize medical care and offer personalized medicine

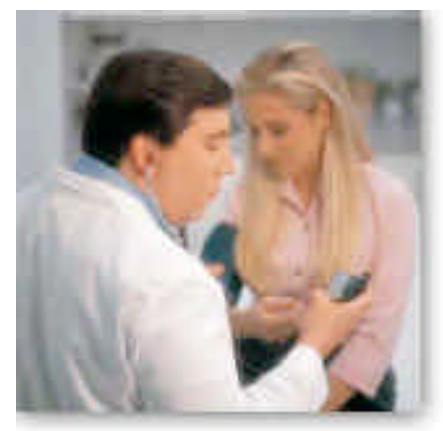

(c) Giovanni De Micheli

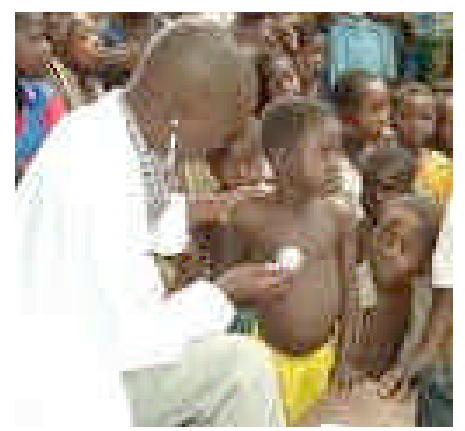




\section{Sample transport}
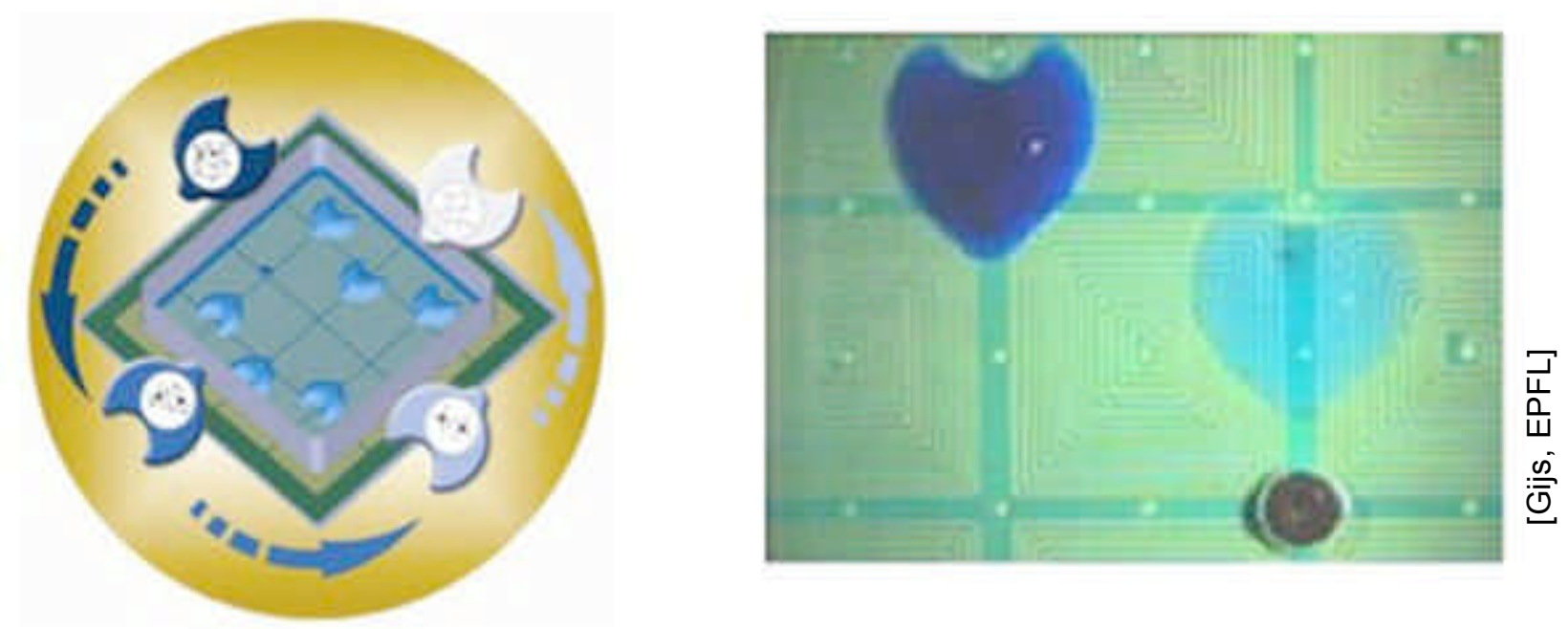

- Cell or sample transport, split and merge

- On a 2-dimensional array

- Parallel scheduling and routing of multiple samples 


\section{Sensing}

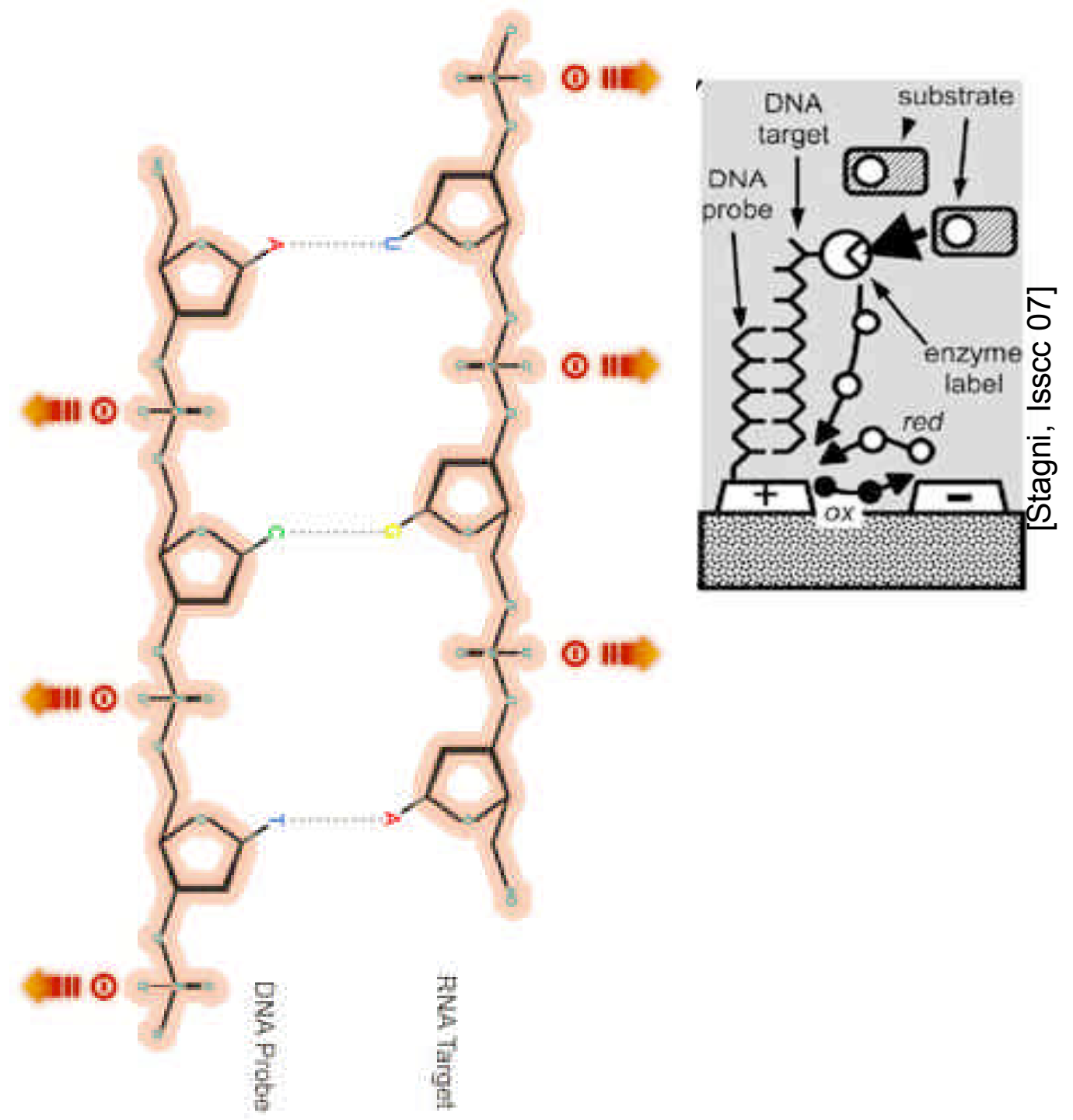

(c) Giovanni De Micheli 


\section{Sensing}

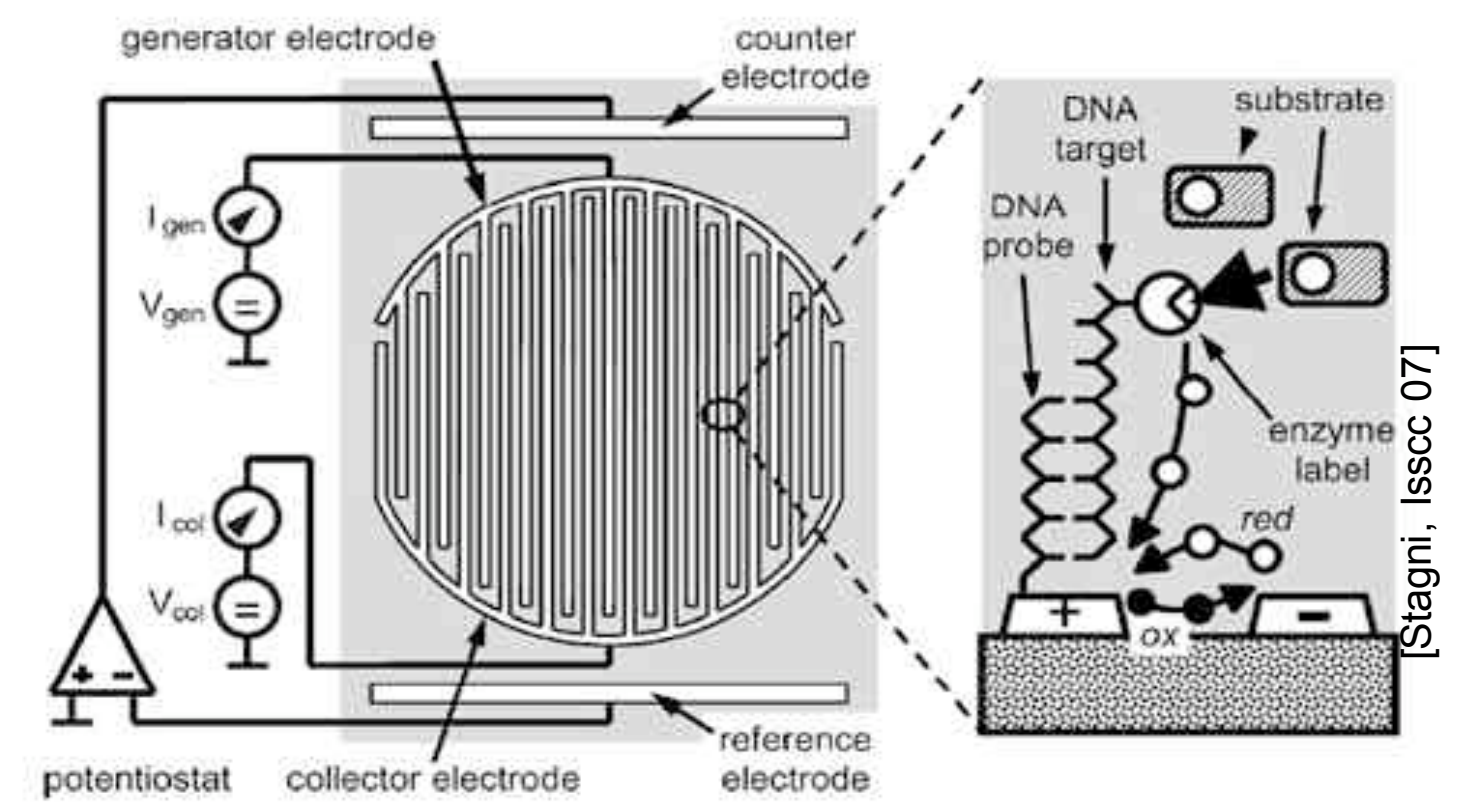

- Non-labelea sensıng tecnnıques are pasea on an electronic reading of hybridization

- Fully integrated system solutions

- Lower cost

- Array detectors yield a matrix of expression levels

(c) Giovanni De Micheli 


\section{Sensing}

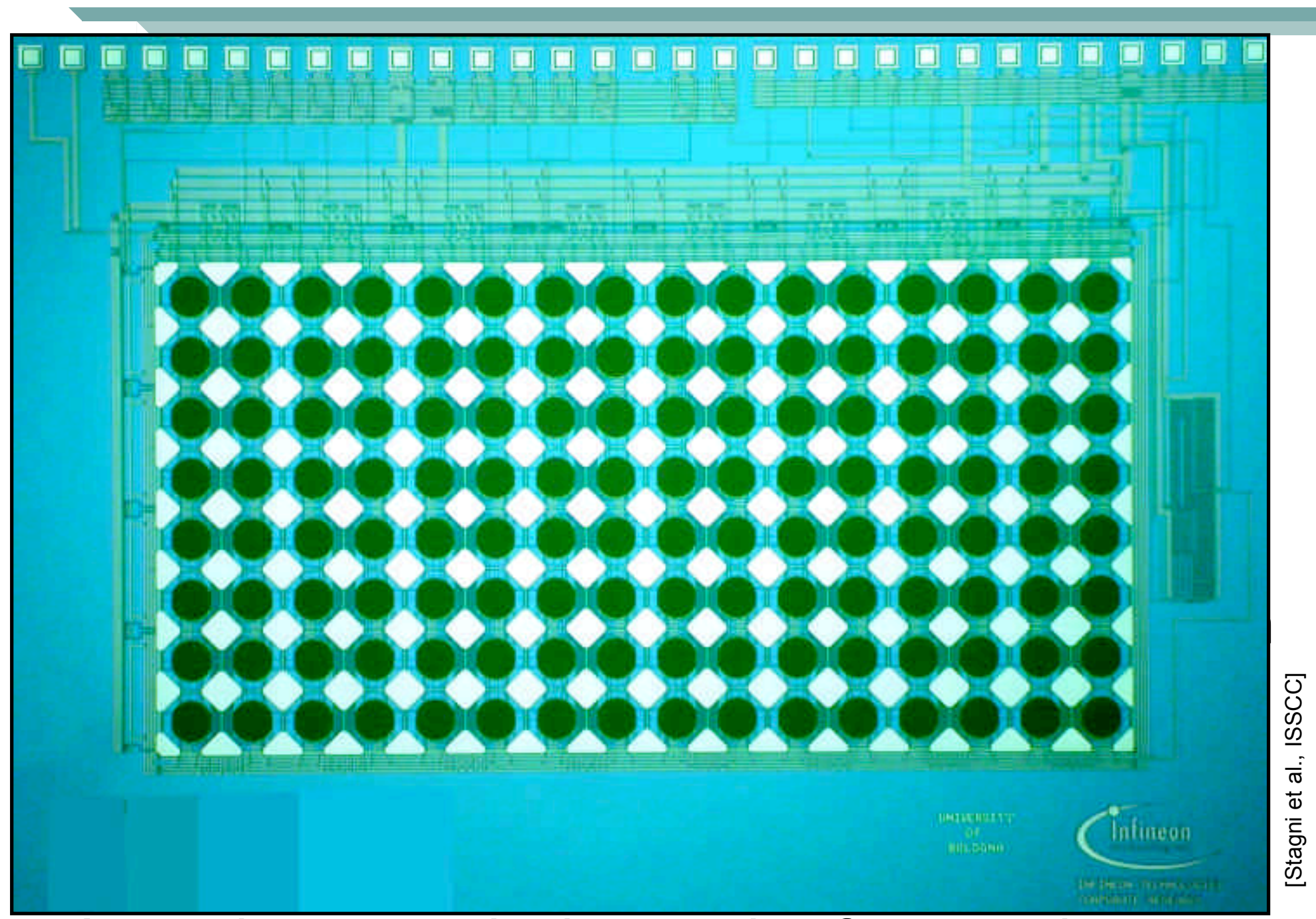

- Array detectors yield a matrix of expression levels

(c) Giovanni De Micheli 


\section{Data mining and interpretation}

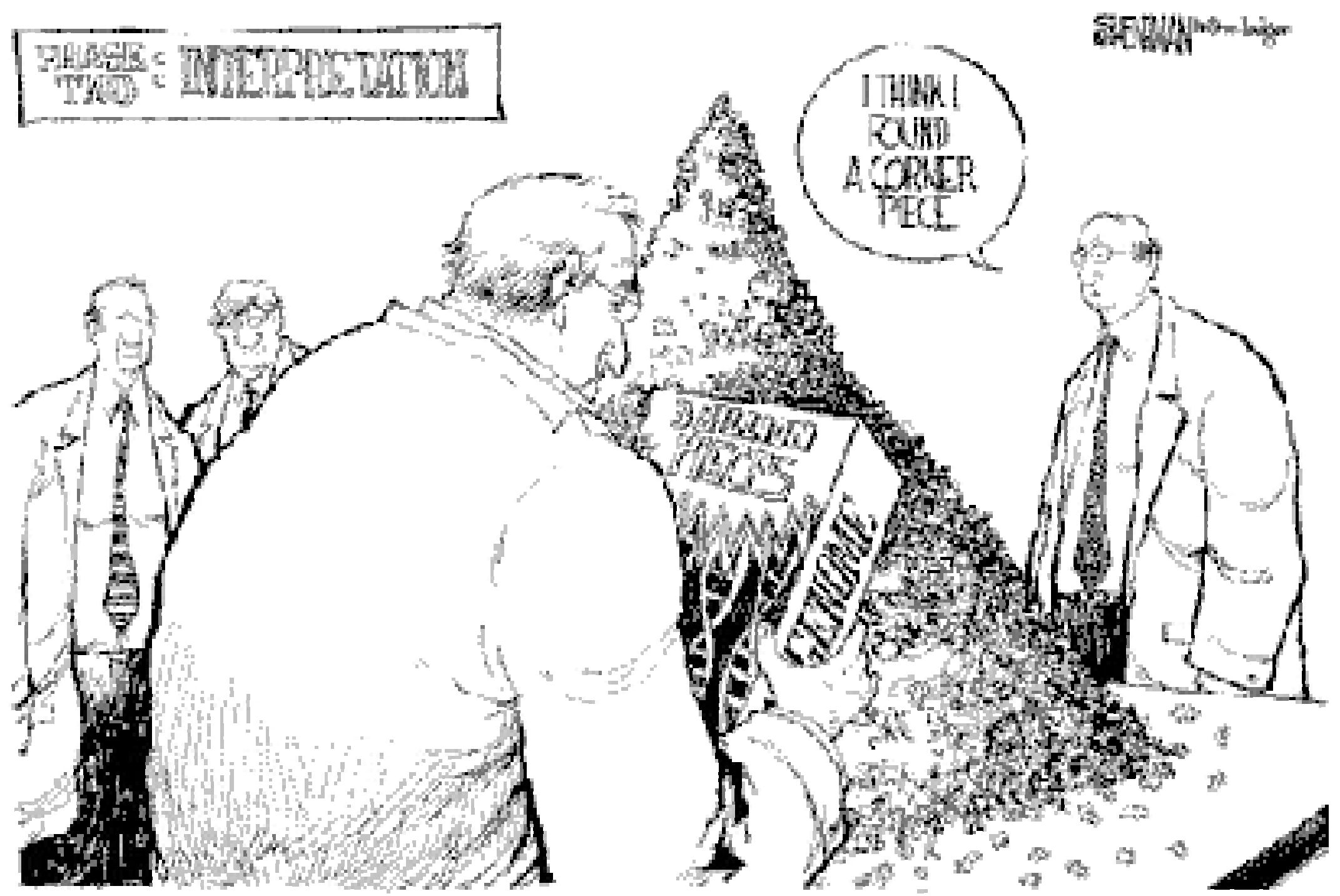

(c) Giovanni De Micheli 


\section{Data interpretation and clustering}

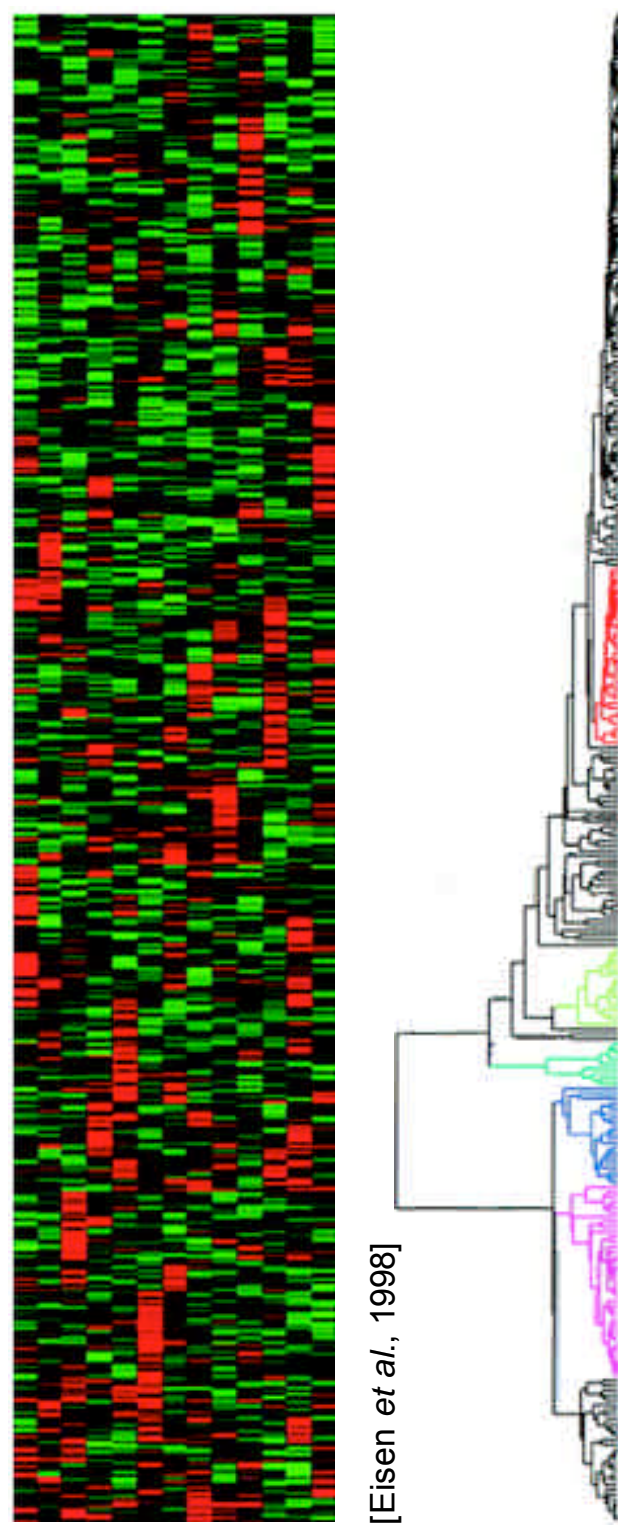

- Grouping similar objects together

- Detecting gene variations consistent with the sample choice

- Inference of specific conditions

- Bi-clustering on large data sets

- Simultaneous cluster of subsets of rows and columns

- Gene and samples

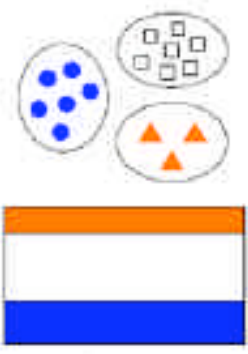

(a) Clestering

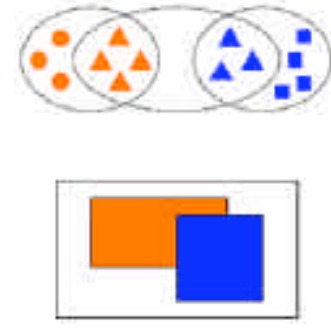

(b) Biclnstaring

- Problem solved with ZDD technology

- Fast and complete data interpretation 


\section{Global Lab on Chip objectives}

- Bio-discovery

- New biological mechanisms

- Medical practice

- Better diagnosis via genetic information

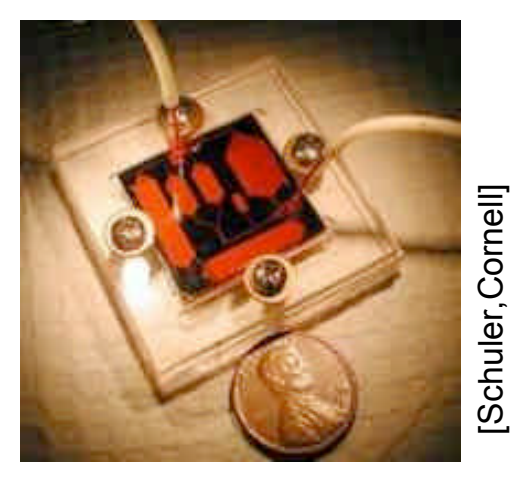

- Linking genetic data to clinical traits and databases

- Micro-chemistry

- Creating organic compounds by micro-reactions

- Support for experiments/tests on the field

- Generic versus application-specific lab on chips

- Programmable, field-programmable? 


\section{Bio analysis and synthesis}

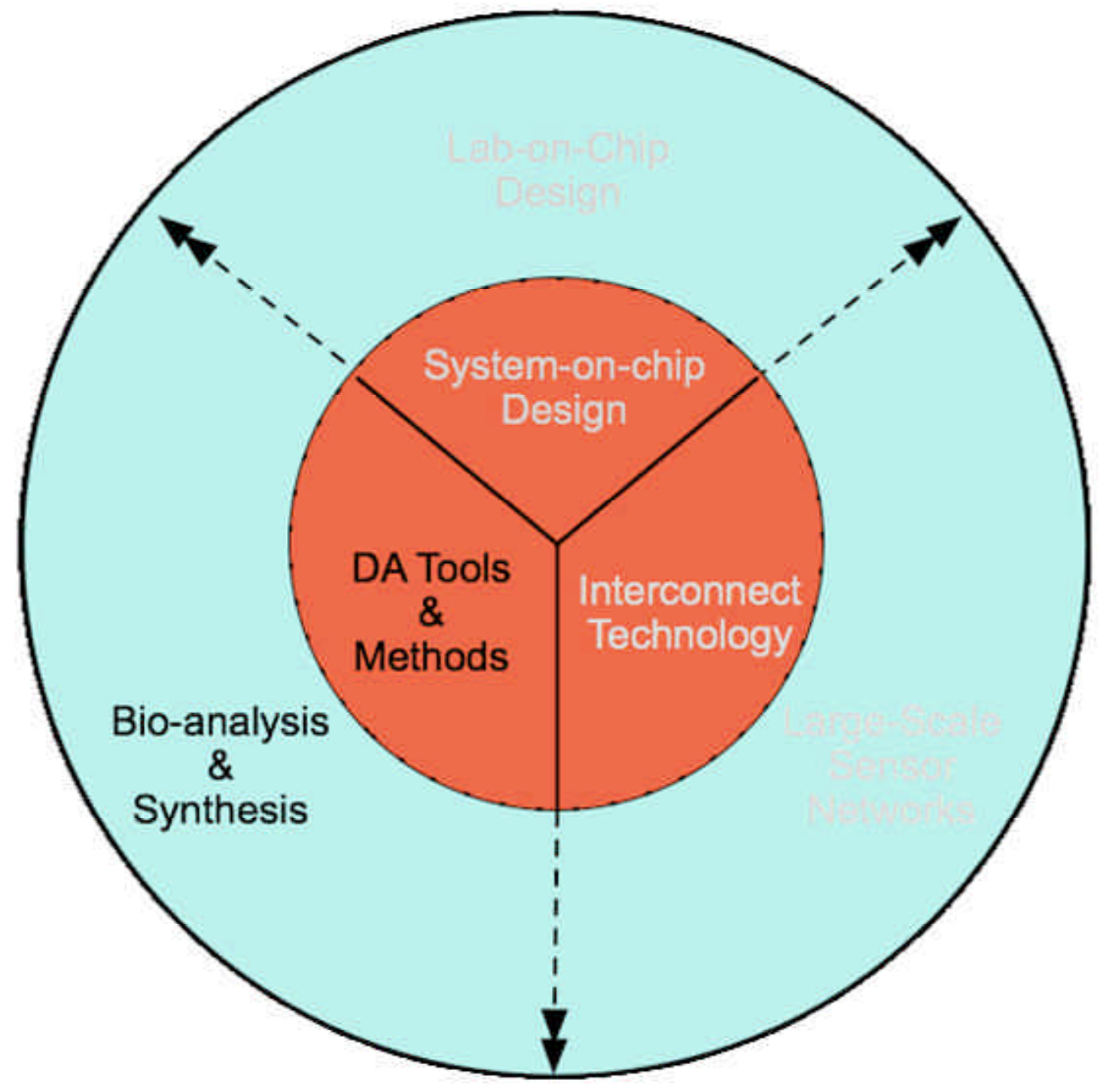




\section{Analysis and synthesis}

- Analysis - understand biological mechanisms

- Comprehend in full the value of the omics

- Genomics, proteinomics, trascriptomics

- Synthesis - modify/create new realities

- Synthesize drugs that alter genetic/metabolic pathways

- Pharmacogenomics

- Synthesize biological compounds that support computation

- Synthetic biology

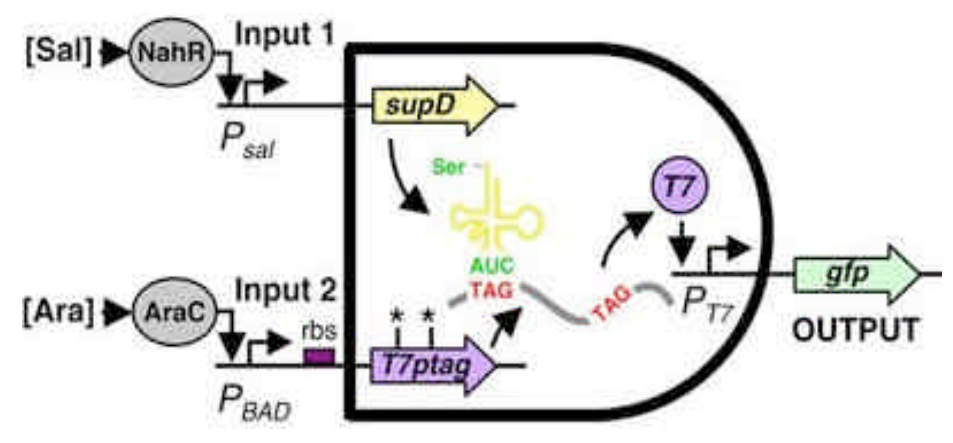

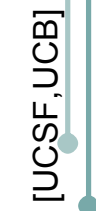

- Multiple abstractions are needed for analysis and synthesis 


\section{Abstractions}

- Bio-chemical abstraction

- Event timing

- Differential equation models

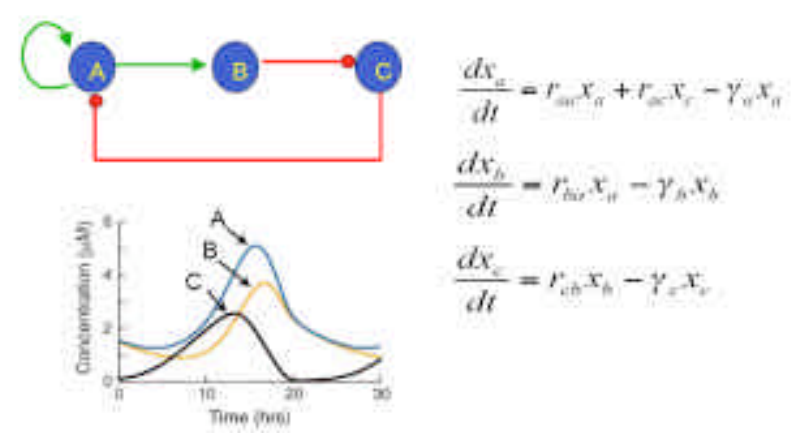

- Logic level abstraction

- Zero-delay model

- Finite-state system

- Synchronous, asynchronous

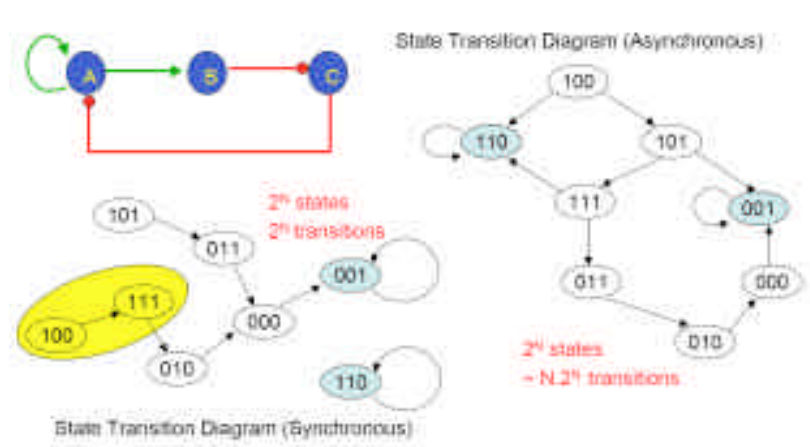

- Functional abstraction

- Biological function

- Input-output analysis

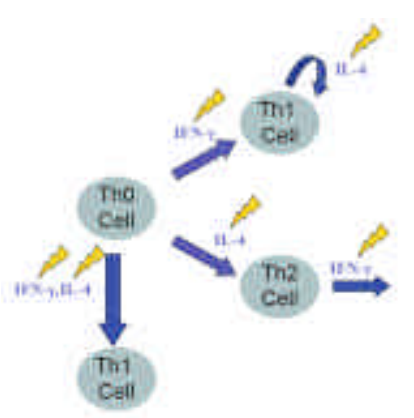

(c) Giovanni De Micheli 


\section{T-helper cells}

- Observed behavior:

- Precursor Th0 cells yield:

- Effector Th1 cells

- Effector Th2 cells

- Evolutions depends on specific gene expressions

- Evolution can be captured by a gene regulatory network

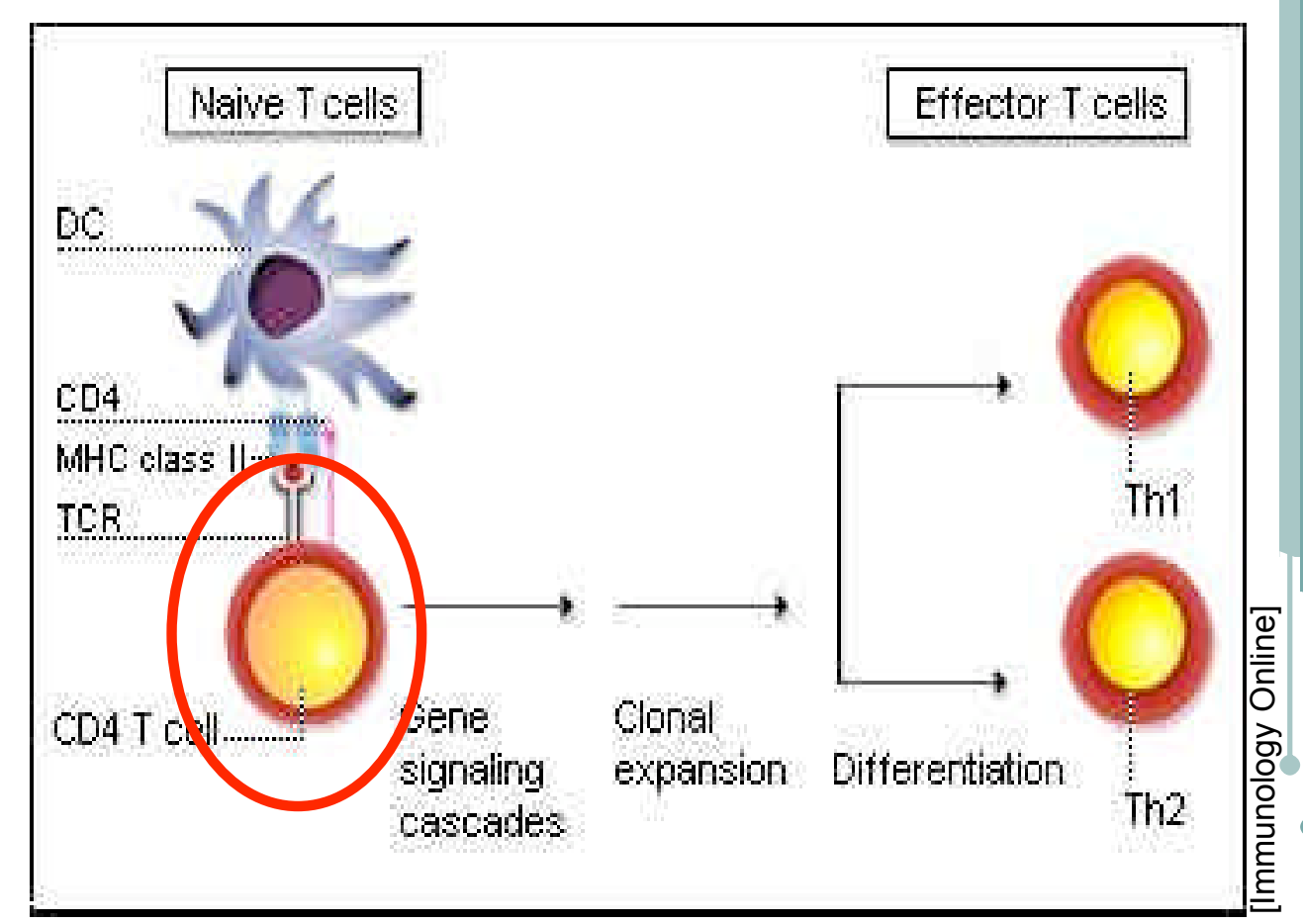

(c) Giovanni De Micheli 


\section{Functional and logic-level model of T-helper cell}

Th0 Cell

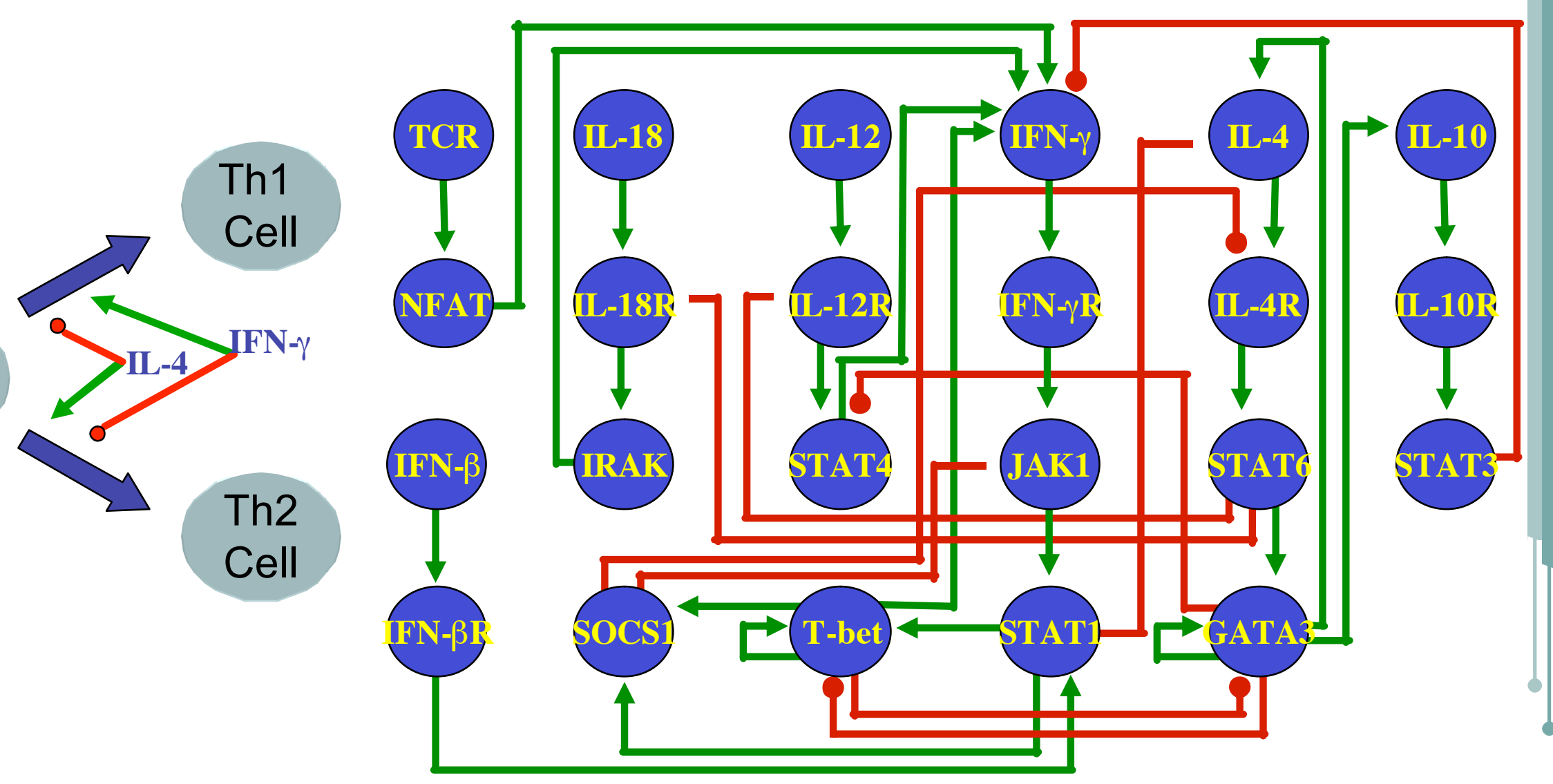




\section{Issues}

- Orthogonalization of concerns

- Focus on terminal behavior independent from timing

- Simulation versus traversal

- Steady state is often the objective

- Implicit methods can handle large amount of data

- Modify system by perturbation

- Knock-out experiment in silico

- Silence a gene

- Stuck-at 0 (déjà vu?) 


\section{Knock-out example: Arabidopsis Thaliana}
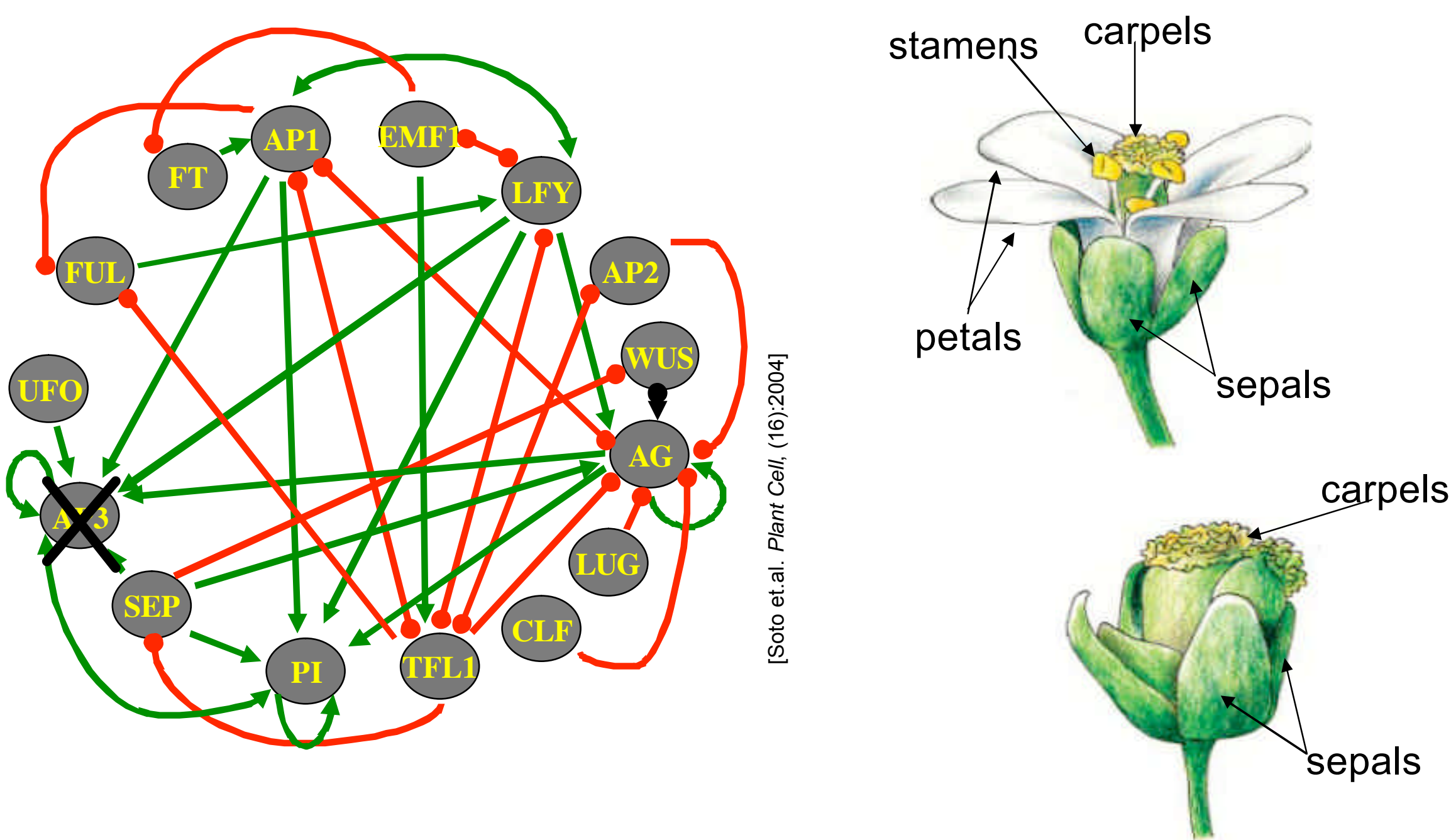

AP3 knockout

(c) Giovanni De Micheli 


\section{Bio analysis \& synthesis objectives}

- Pharmacogenomics

- Develop drug therapy, cognizant of patient genotype

- Study effects of altering genetic/metabolic pathways

- Synthetic biology

- Engineer systems based on biological components

- Abstraction: libraries, synthesis process

- Biology-driven computation

- Devise computational processes performed by DNA

- Biologic scaffolding

- Construct nano structures/circuits using DNA composition

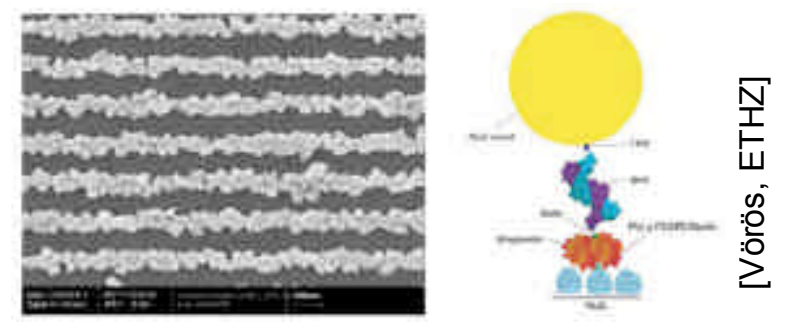




\section{Environmental monitoring and control}

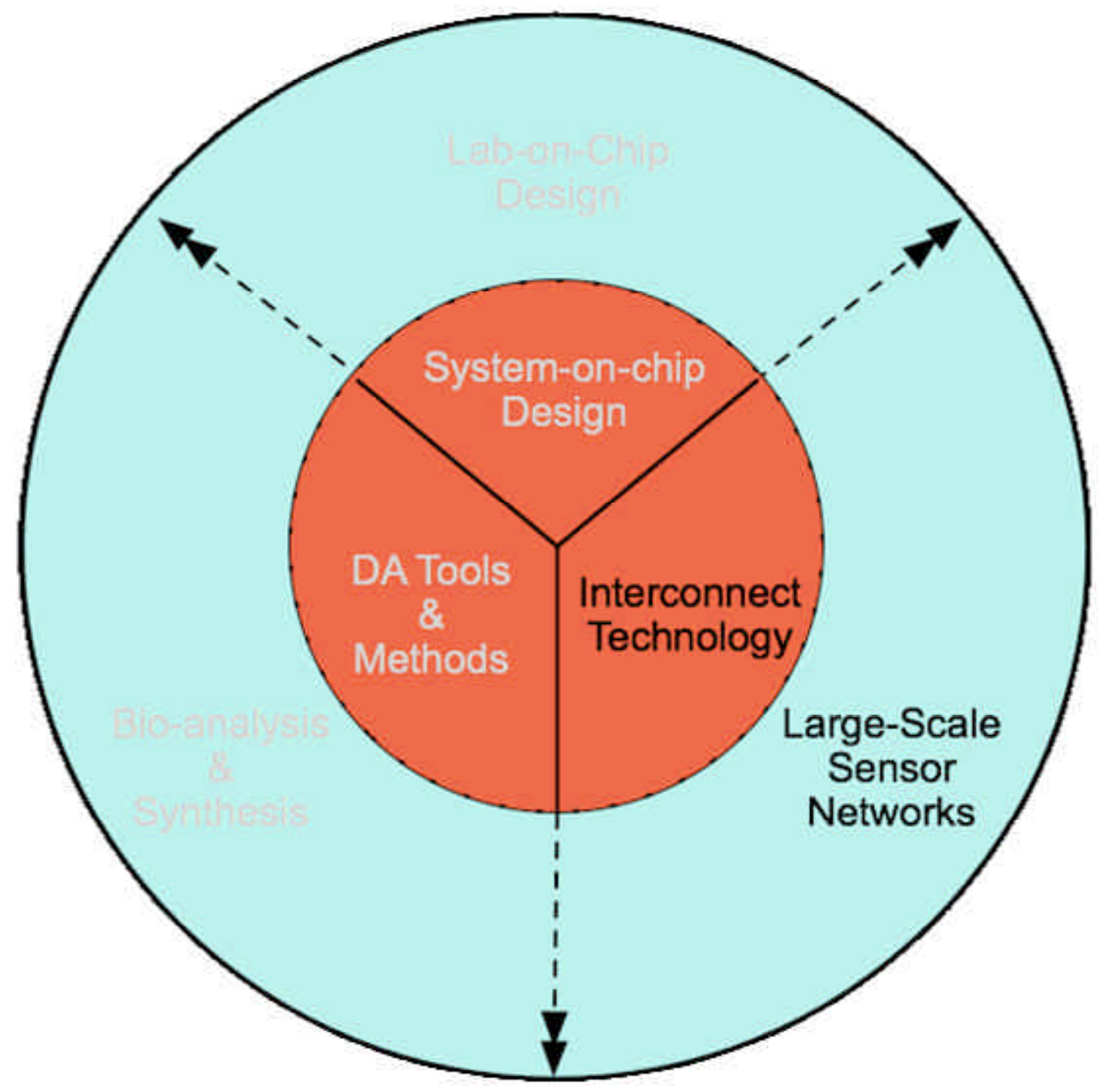




\section{The environment}

- We are embedded in the environment

- Many inconvenient truths
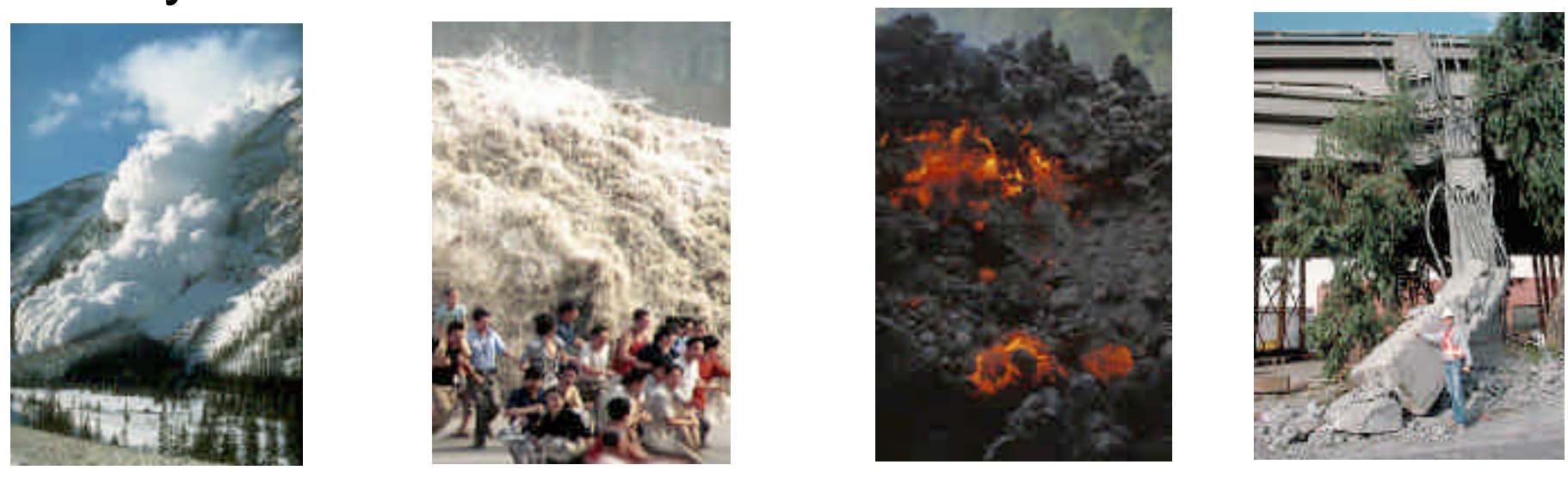

- What are the challenges of wireless sensor networks to monitor/control the environment?

- Massive amount of data to process

- Distributing and powering the nodes

- Providing redundancy to tolerate local failures 


\section{Engineering environmental systems}

- Integrated sensing, computation, communication and embedded software

- Local vs. global data processing and communication

- The power of data abstraction

- Data reduction and integration

- The distributed intelligence approach

- Reason and act locally with (some) global information

- New computational paradigms, as compared to classical supercomputer approaches
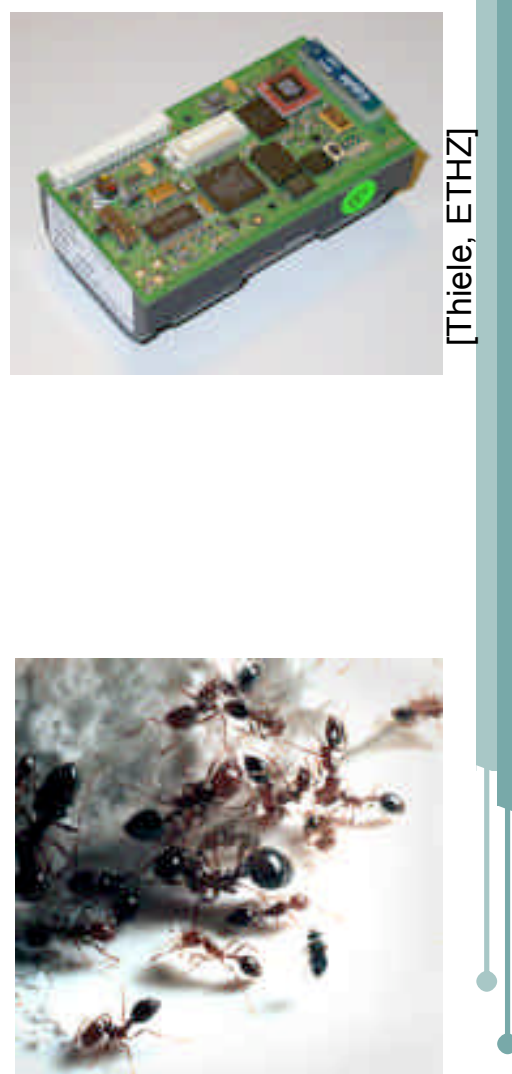


\section{The quest for energy efficiency}

- Distributed wireless systems must (eventually) be autonomous

- Energy harvesting from the environment

- Mobile and fixed applications

- Convert unused (degraded) energy into information

- Energy distribution systems must be efficient

- Use information on the system to optimize energy distribution

- Smart home, building, factory, ...

- Electric grid management

- Convert information into energy savings

- Mutual interaction: energy ? information

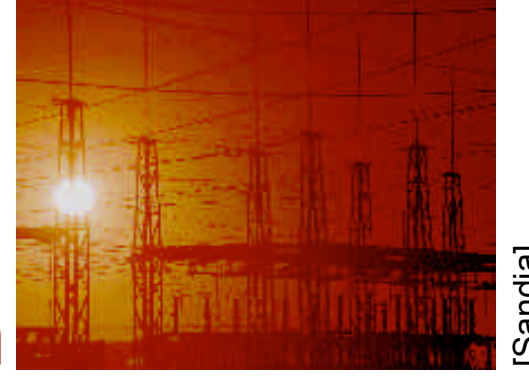

- Policies for run-time energy/information management will play key role in system design 


\section{How do we interact with the environment?}

- Physically

- Socially
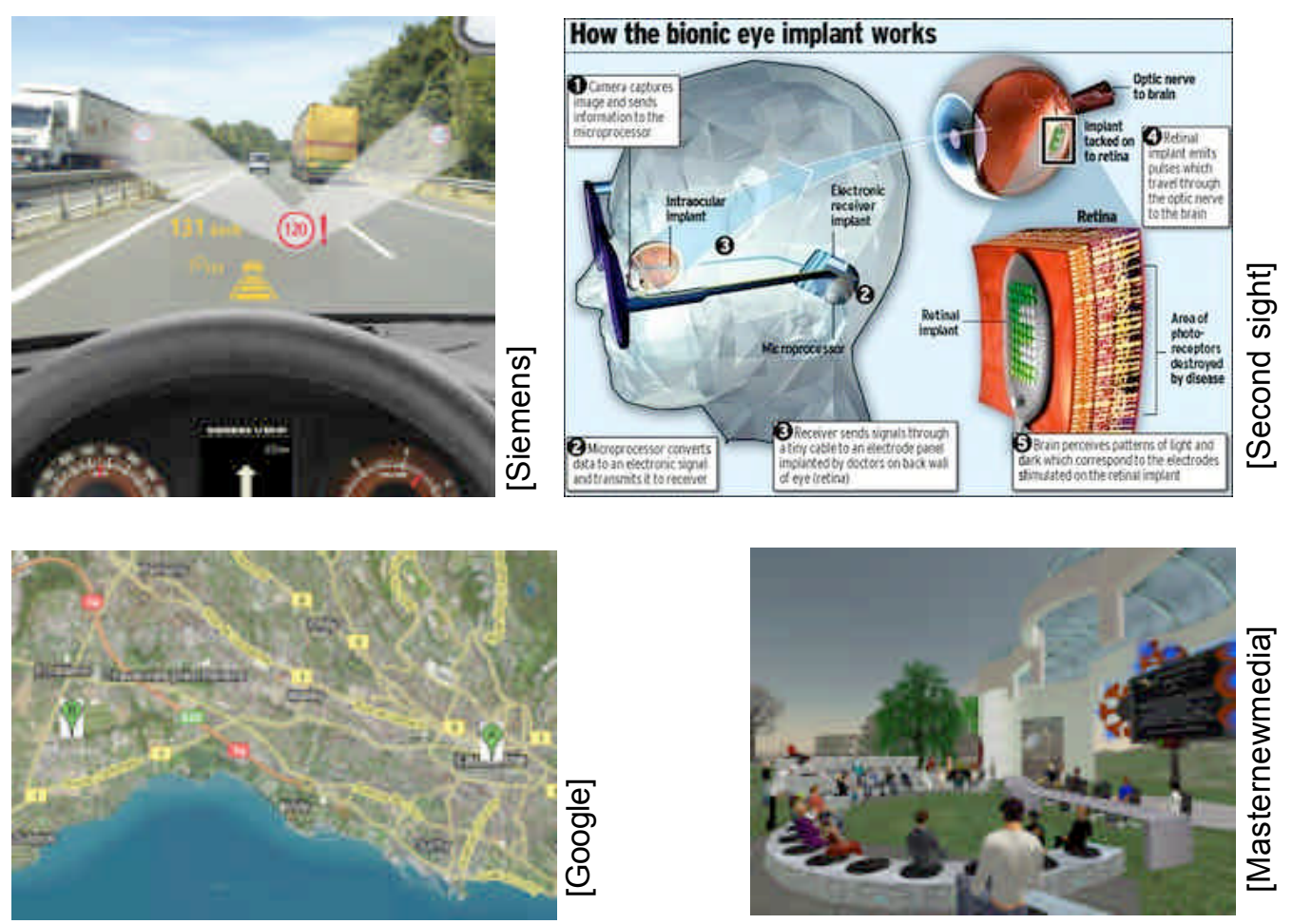

- How to design evolvable embedded environments with user interaction and immersion? 


\section{How do we interact with the environment?}

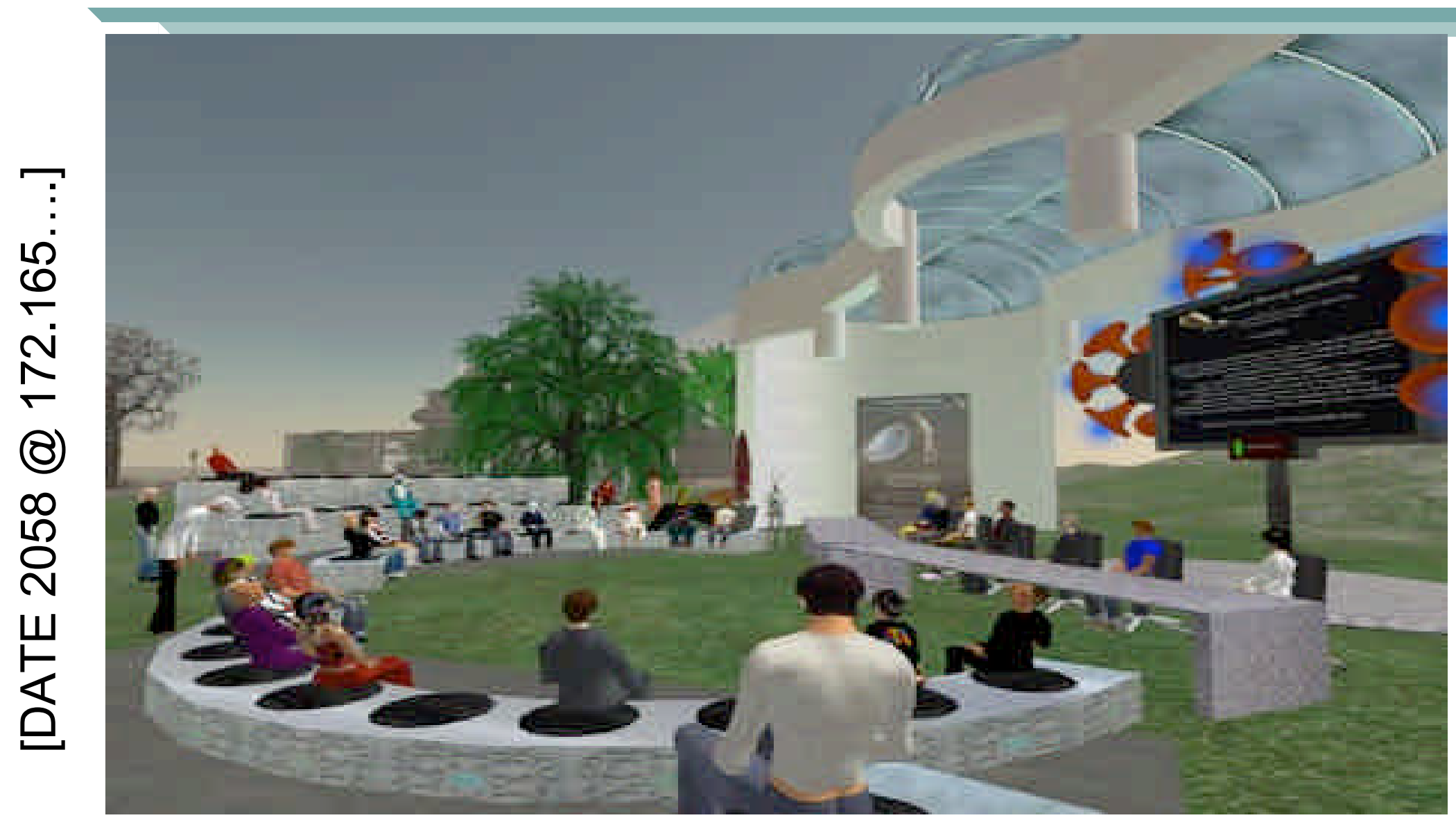

- How to design evolvable embedded environments with user interaction and immersion? 


\section{Cooperative engineering}

- Bringing together engineer/scientists/doctors with different skills

- Communication and vocabulary

- Abstraction and modularity

- Collaborative workspaces
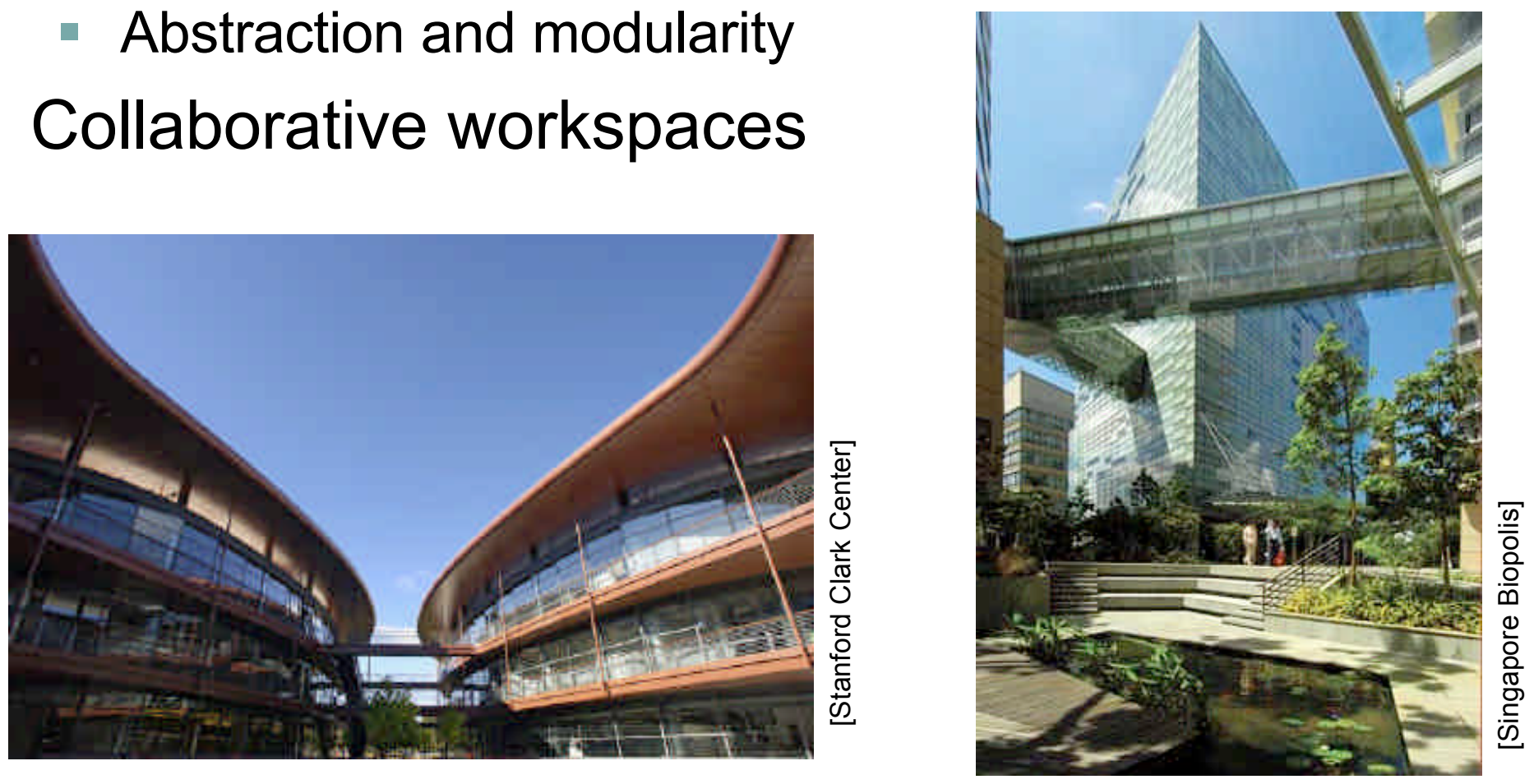

(c) Giovanni De Micheli 


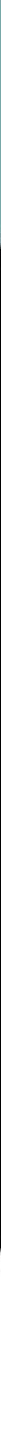

(c) Giovanni De Micheli 


\section{The humanitarian technology challenge}

\section{\. IEEE}
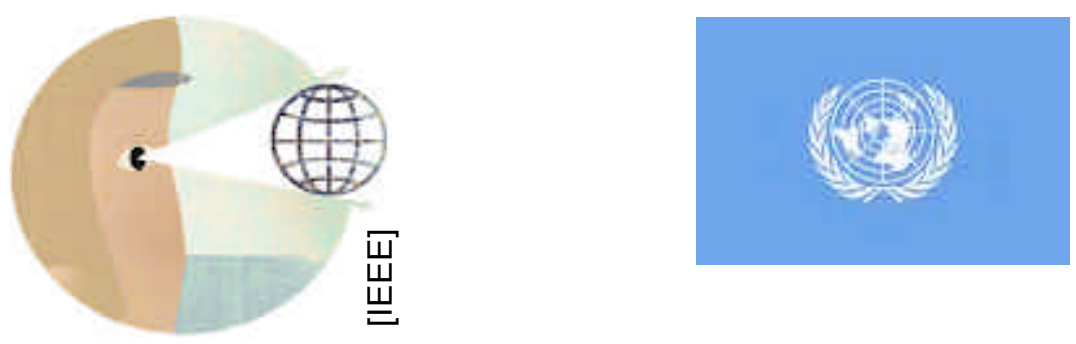

- A new partnership between the IEEE and the UN

- Identify technologies in the health/environment domain that can benefit developing countries

- Food, water, health monitoring

- Using cellular technology to link data

- Ubiquitous connectivity and local data processing

- Autonomous or very low-power consumption because of limited availability of energy

- An ethical objective that can raise enthusiasm among engineers 


\section{Summary}

- The road ahead has challenges and rewards:

- Expanding our horizon is key to scientific viability and commercial profitability

- We need heterogeneous hardware design and the corresponding software infrastructure

- Product/system design is an extremely complex task, because of the variety of facets and technologies involved

- System-level design technologies are crucial for system conception, design and management

- Progress leads us beyond advanced silicon chip design

- Scientific and financial benefits will stem from the system/service perspective 


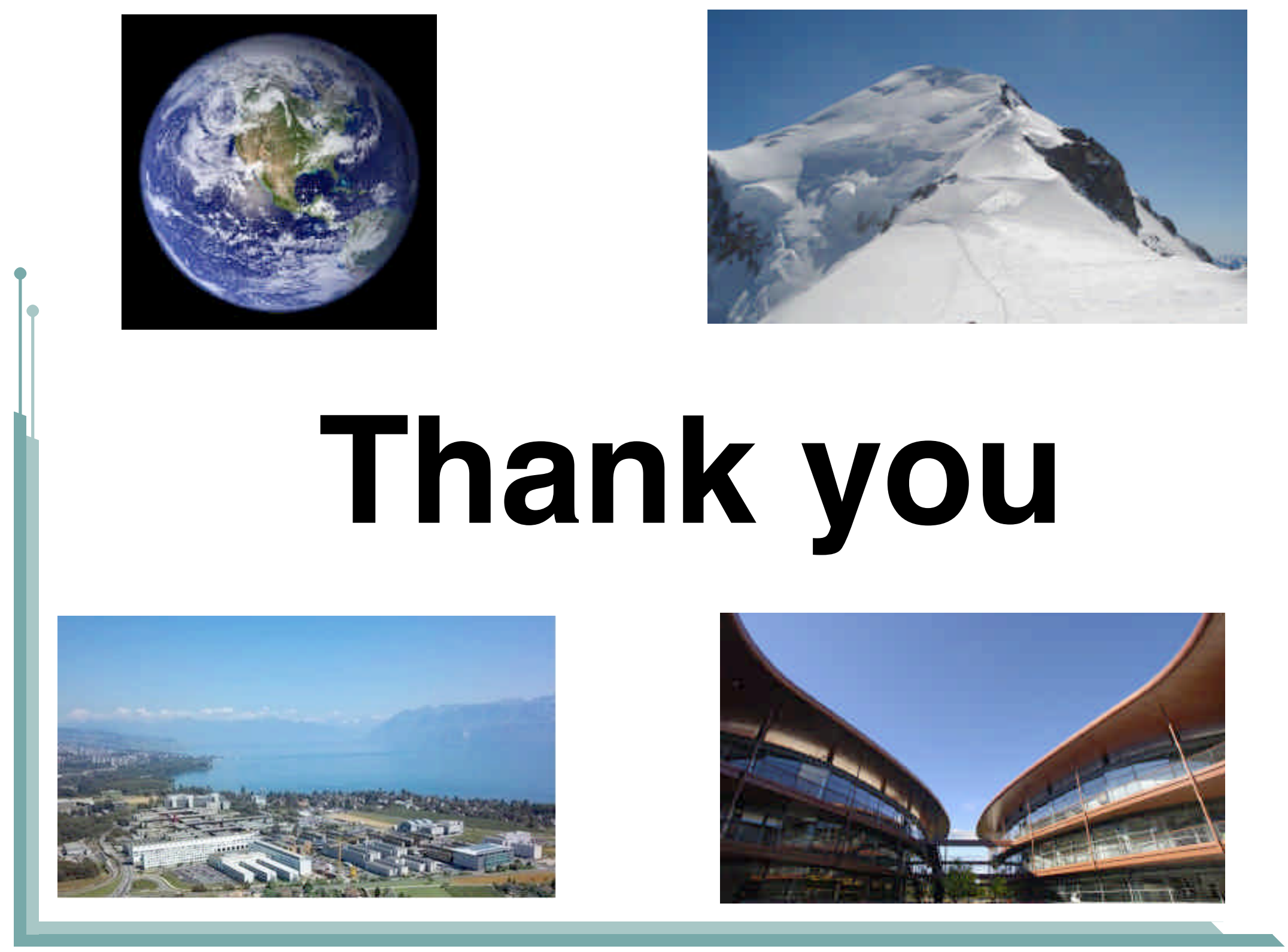

